\title{
A D-induced duality and its applications
}

\author{
Jan Brinkhuis · Shuzhong Zhang
}

Received: 26 July 2004 / Accepted: 18 December 2006 / Published online: 30 March 2007 (C) Springer-Verlag 2007

\begin{abstract}
This paper attempts to extend the notion of duality for convex cones, by basing it on a prescribed conic ordering and a fixed bilinear mapping. This is an extension of the standard definition of dual cones, in the sense that the nonnegativity of the inner-product is replaced by a pre-specified conic ordering, defined by a convex cone $\mathrm{D}$, and the inner-product itself is replaced by a general multi-dimensional bilinear mapping. This new type of duality is termed the D-induced duality in the paper. We further introduce the notion of D-induced polar sets within the same framework, which can be viewed as a generalization of the D-induced dual cones and is convenient to use for some practical applications. Properties of the extended duality, including the extended bi-polar theorem, are proven. Furthermore, attention is paid to the computation and approximation of the D-induced dual objects. We discuss, as examples, applications of the newly introduced D-induced duality concepts in robust conic optimization and the duality theory for multi-objective conic optimization.
\end{abstract}

Keywords Convex cones · Duality · Duality theorem - Conic optimization Mathematics Subject Classification (2000) $\quad 46 \mathrm{~A} 20 \cdot 46 \mathrm{~N} 10 \cdot 90 \mathrm{C} 25$

Research supported in part by the Foundation 'Vereniging Trustfonds Erasmus Universiteit Rotterdam' in The Netherlands, and in part by Hong Kong RGC Earmarked Grants CUHK4174/03E and CUHK418406.

\footnotetext{
J. Brinkhuis $(\varangle)$

Econometric Institute, Erasmus University, Rotterdam, The Netherlands e-mail: brinkhuis@few.eur.nl

S. Zhang

Department of Systems Engineering and Engineering Management, The Chinese University of Hong Kong, Shatin, Hong Kong
} 


\section{Introduction}

Duality plays a central role in the development of the theory as well as the solution methods for optimization. A good example is the success of the so-called primal-dual interior point methods for conic convex optimization; see, e.g., [10].

A dual object, say the dual of a convex cone, is defined as the set of all nonnegative linear mappings (functionals) over the cone under consideration. This set itself forms a convex cone and has an intimate and interesting relationship with the original cone. Duality theory is devoted to revealing the nature of the relationship, and beyond any doubt it has become the foundation of optimization. However, there are circumstances where the concept of nonnegativity needs to be extended in order to better suit some new and interesting applications, arising, e.g., from robust analysis of conic optimization. In this paper we introduce a new type of duality for convex cones, where the nonnegativity is induced by an arbitrary given convex cone, which is obviously a generalization of the usual definition of the dual. This usual definition is the special case that the given convex cone is the nonnegative half-line $\Re_{+}$. We show that under some conditions, important results such as the duality theorem can be carried over. A key issue is the characterization of the new kind of dual cone, termed as the D-induced dual cone in this paper. It is linked naturally to other important issues in convex analysis. For instance, it raises questions such as how to compute the tensor product of two convex cones, and what is the calculus rule for the duality operation (in the ordinary sense) for the tensor product of two convex cones. We believe that this triggers interesting research questions to be answered in the future. The concept is further generalized to the setting of a D-induced polar set. Although a D-induced polar set can be viewed as an inhomogeneous version of the D-induced cone, its direct form is handy to use for practical purposes, and it has interesting properties of its own. Applications arising from robust optimization and multi-objective optimization are discussed. We believe the potentials for the $\mathrm{D}$-induced duality abound. As a key issue we point out that it is crucial to study the approximative computation of a D-induced dual object, as the exact computation is often NP-hard.

This paper is organized as follows. We shall introduce the new type of duality in Sect. 2. In the same section we show several properties of the new duality operation, including the extended duality theorem and several calculus rules of the new duality operation. In Sect. 3 we discuss the related polar operations and their properties. Section 4 is concerned with how to compute and approximate the new dual objects. In Sect. 5, we discuss two applications, one from the robust version of conic convex optimization, and the other from multiple objective conic convex optimization. Then, in Sect. 6 we discuss how the new type of duality can be characterized and computed from a totally different point of view: the epigraph representation of convex cones. Finally, we conclude the paper in Sect. 7.

Notations: In most places, letters in calligraphic style, e.g. $\mathcal{X}$, denote vector spaces; $\Re^{n}$ is $n$-dimensional Euclidean space; $\Re_{+}^{n}$ is the set of all $n$-dimensional 
non-negative vectors; $\Re_{++}^{n}$ is the set of all $n$-dimensional positive vectors; $\mathcal{S}^{m}$ is the space of all $m$ by $m$ symmetric real-valued matrices; $\mathcal{S}_{+}^{m}$ is the set of all $m$ by $m$ positive semidefinite matrices $\left(X \in \mathcal{S}_{+}^{m}\right.$ is equivalent to $\left.X \succeq 0\right) ; \mathcal{S}_{++}^{m}$ is the set of all $m$ by $m$ positive definite matrices; $\|\cdot\|$ is the Euclidean norm with appropriate dimension from the context; cl $S$ stands for the closure of the set $S$; conv $S$ stands for the convex hull of the set $S ; S_{1}+S_{2}$ denotes the sum of two sets $S_{1}$ and $S_{2}$; epi $(f)$ stands for the epigraph of the function $f ; \operatorname{SOC}(n)$ is the standard $n$-dimen-

sional second order cone, i.e., $\operatorname{SOC}(n)=\left\{\left[x_{1}, x_{2}, \ldots, x_{n}\right]^{T} \mid x_{1} \geq \sqrt{\sum_{i=2}^{n} x_{i}^{2}}\right\}$; $e_{i}^{(n)}$ indicates the $i$ th unit vector in $\Re^{n}$, namely the vector whose $i$ th component is 1 while all other components are 0 . Finally, $\operatorname{vec}(A)$ stands for the vector obtained by stacking together the columns of the matrix $A$, i.e., vec $(A)=$ $\left[a_{1}^{T}, a_{2}^{T}, \ldots, a_{m}^{T}\right]^{T}$ where $\left[a_{1}, a_{2}, \ldots, a_{m}\right]=A$.

\section{The D-induced dual cone}

Consider three vector spaces $\mathcal{X}, \mathcal{Y}$, and $\mathcal{W}$.

Let $\mathrm{D} \subseteq \mathcal{W}$ be a certain fixed convex cone. We assume that $\mathrm{D}$ is not a linear subspace; this is equivalent to demanding that there is $d \in \mathrm{D}$ such that $-d \notin \mathrm{D}$. This will be called a non-flat cone. Due to the convexity of D, by a separation argument this condition further implies the existence of $d \in \mathrm{D}$ such that $-d \notin \mathrm{cl} \mathrm{D}$; this will be called a non-flat direction.

Let

$$
\langle x, y\rangle:(x, y) \in \mathcal{X} \times \mathcal{Y} \rightarrow \mathcal{W}
$$

be a given bilinear mapping, i.e., for any fixed $x \in \mathcal{X},\langle x, \cdot\rangle: \mathcal{Y} \rightarrow \mathcal{W}$ is a linear mapping, and for any fixed $y \in \mathcal{Y},\langle\cdot, y\rangle: \mathcal{X} \rightarrow \mathcal{W}$ is a linear mapping as well.

In this paper, we assume throughout, for the sake of simplicity, that all vector spaces under consideration are finite dimensional, although some of the results can be extended easily to a more general setting. Moreover, we choose for each vector space an inner product: we denote the chosen inner product on $\mathcal{X}$ by $\langle\cdot, \cdot\rangle_{\mathcal{X}}$; similarly for $\mathcal{Y}$ and $\mathcal{W}$. In particular, we assume after suitable choices of coordinates that $\mathcal{X}=\Re^{n}, \mathcal{Y}=\Re^{m}$, and $\mathcal{W}=\Re^{k}$ and that the inner products are each the usual inner product, viz. the sum of coordinate-wise products, e.g., $\left\langle x^{1}, x^{2}\right\rangle_{\mathcal{X}}=\left(x^{1}\right)^{T} x^{2}$ for $x^{1}, x^{2} \in \mathcal{X}$. In our applications we consider often spaces of matrices; here the choice of coordinates means that we stack the matrices into column-vectors as described before.

Let $U \subseteq \mathcal{X}$ be a cone, not necessarily convex.

The dual of $U$, induced by $\mathrm{D}$ under $\langle\cdot, \cdot\rangle$, is a convex cone in $\mathcal{Y}$, defined by

$$
\begin{aligned}
U_{\mathrm{D}}^{*} & =\{y \in \mathcal{Y} \mid\langle x, y\rangle \in \mathrm{D} \text { for all } x \in U\} \\
& =\{y \in \mathcal{Y} \mid\langle U, y\rangle \subseteq \mathrm{D}\} .
\end{aligned}
$$


In other words, the D-induced dual cone of $U$ is the collection of all $y$ for which the linear mapping $x \rightarrow\langle x, y\rangle$ takes $U$ to $\mathrm{D}$.

Analogously, for a cone $V$ in the space $\mathcal{Y}$, its $\mathrm{D}$-induced dual under the bilinear mapping $\langle\cdot, \cdot\rangle$ is a convex cone in $\mathcal{X}$, defined by

$$
\begin{aligned}
V_{\mathrm{D}}^{*} & =\{x \in \mathcal{X} \mid\langle x, y\rangle \in \mathrm{D} \text { for all } y \in V\} \\
& =\{x \in \mathcal{X} \mid\langle x, V\rangle \subseteq \mathrm{D}\} .
\end{aligned}
$$

We emphasize that there are two key factors in this definition, to wit the orderdefining cone $\mathrm{D}$ and the bilinear mapping $\langle x, y\rangle:(x, y) \in \mathcal{X} \times \mathcal{Y} \rightarrow \mathcal{W}$.

When we speak of the D-induced dual of a cone, it is of importance to specify in which of the two spaces, $\mathcal{X}$ or $\mathcal{Y}$, the cone in question resides; then the $\mathrm{D}$-induced dual lies in the other space. If we choose $\mathcal{W}=\Re, \mathrm{D}=\mathfrak{R}^{+}, \mathcal{Y}=\mathcal{X}$, and $\langle\cdot, \cdot\rangle$ an inner product on $\mathcal{X}$, we get the dual in the ordinary sense. Then, due to the fact that the inner product is a symmetric bilinear pairing, one does not have to specify whether the given cone is contained in $\mathcal{X}$ or in $\mathcal{Y}$.

We note that since $\mathrm{D}$, being a cone, contains the origin, any $\mathrm{D}$-induced dual cone must contain the origin as well. In particular, it is non-empty. Obviously, $U_{\mathrm{D}}^{*}$ is closed if $\mathrm{D}$ is closed. However, we do not require $\mathrm{D}$ to be closed. A cone in $\mathcal{W}$ has the same closure as $\mathrm{D}$ if it is contained in cl $\mathrm{D}$ and, moreover, contains the convex cone relint $t_{0} D:=$ relint $D \cup\{0\}$. The closure of the $D$-dual of a cone depends only on the closure of $\mathrm{D}$. This property is formalized in the following proposition.

Proposition 2.1 Let $\mathrm{D}$ be a convex cone. Let $U \subseteq \mathcal{X}$ be a cone. It holds that

$$
\operatorname{cl} U_{\text {relint }_{0} \mathrm{D}}^{*}=\operatorname{cl} U_{\mathrm{D}}^{*}=U_{\mathrm{cl} \mathrm{D}}^{*}
$$

Proof Since relint 0 D $\subseteq \mathrm{D} \subseteq \mathrm{cl} \mathrm{D}$, we have cl $U_{\text {relint }_{0} \mathrm{D}}^{*} \subseteq \operatorname{cl} U_{\mathrm{D}}^{*} \subseteq U_{\text {cl D }}^{*}$

We shall now show that $U_{\mathrm{cl} \mathrm{D}}^{*} \subseteq \operatorname{cl} U_{\text {relint }_{0} \mathrm{D}}^{*}$, from which the desired result follows. Suppose by contradiction that there is $y \in U_{\mathrm{cl} \mathrm{D}}^{*} \backslash \mathrm{cl} U_{\text {relint }_{0} \mathrm{D}}^{*}$. Let $\bar{y}$ be the projection of $y$ on $\mathrm{cl} U_{\text {relint }_{0} \mathrm{D}}^{*}$. Let $\delta=\|y-\bar{y}\|>0$. Choose a sequence $\left\{\bar{y}_{n} \mid n=1,2, \ldots\right\} \subseteq U_{\text {relint }_{0} \mathrm{D}}^{*}$ such that $\lim _{n \rightarrow \infty} \bar{y}_{n}=\bar{y}$. Therefore, for any $u \in U$ we have $\left\langle u, \bar{y}_{n}\right\rangle \in$ relint $_{0} \mathrm{D}$, and, because $y \in U_{\mathrm{cl} \mathrm{D}}^{*}$ we have $\langle u, y\rangle \in \mathrm{cl} \mathrm{D}$. Since $\mathrm{D}$ is a convex cone, it follows that $\left\langle u,\left(y+\bar{y}_{n}\right) / 2\right\rangle \in \operatorname{relint}_{0} \mathrm{D}$ for any $u \in U$. That is, $\left(y+\bar{y}_{n}\right) / 2 \in U_{\text {relint }_{0} \mathrm{D}}^{*}$. Consequently, by taking the limit on $n$ we obtain that $(y+\bar{y}) / 2 \in \mathrm{cl}_{\text {relint }_{0} \mathrm{D}}^{*}$. At the same time, we have $\|y-(y+\bar{y}) / 2\|=\delta / 2$, which contradicts with the fact that $\bar{y}$ is the projection of $y$ on $\mathrm{cl} U_{\text {relint }_{0} \mathrm{D}}^{*}$. This contradiction shows that the proposition holds.

A key result concerning the D-induced duality is the extended duality theorem. Before presenting this result, let us first introduce the following notion of surjectivity. 
Linear functions and bilinear mappings can be described in terms of coordinates as follows. For each linear function on a vector space $\mathcal{X}$ endowed with an inner-product $\langle\cdot, \cdot\rangle_{\mathcal{X}}$ there is a unique $a \in \mathcal{X}$ such that the linear function can be written as $x \mapsto\langle a, x\rangle_{\mathcal{X}}$. Each bilinear mapping $\langle x, y\rangle:(x, y) \in \mathcal{X} \times \mathcal{Y} \rightarrow$ $\mathcal{W}$, can be written in the following form, using an appropriate coordinate system:

$$
\langle x, y\rangle=\left[\begin{array}{c}
x^{T} A_{1} y \\
\vdots \\
x^{T} A_{k} y
\end{array}\right],
$$

where $A_{i} \in \Re^{n \times m}, i=1, \ldots, k$.

Definition 2.2 Consider the bilinear mapping

$$
\langle x, y\rangle:=\left[\begin{array}{c}
x^{T} A_{1} y \\
\vdots \\
x^{T} A_{k} y
\end{array}\right]:(x, y) \in \mathcal{X} \times \mathcal{Y} \rightarrow \mathcal{W} .
$$

We call $\langle\cdot, \cdot\rangle$ dual surjective with respect to $D$ if for any $a \in \mathcal{X}$ there is a non-flat direction $b \in \mathrm{D}(-b \notin \mathrm{cl} \mathrm{D})$ and an element $y \in \mathcal{Y}$ such that the linear matrix equation

$$
\left[A_{1} y, \ldots, A_{k} y\right]=a b^{T}
$$

is satisfied. This concept does not depend on the choice of coordinates as can be seen from the following coordinate-free description: $\langle\cdot, \cdot\rangle$ is dual surjective with respect to $D$ if and only if

$$
\forall a \in \mathcal{X}, \exists \text { non-flat } b \in \mathrm{D}, \exists y \in Y \text {, such that }\langle x, y\rangle=\langle x, a\rangle_{\mathcal{X}} b \text { for all } x \in \mathcal{X} \text {. }
$$

Similarly, we call $\langle\cdot, \cdot\rangle$ primal surjective with respect to $D$ if for any $d \in \mathcal{Y}$ there is a non-flat direction $c \in \mathrm{D}(-c \notin \mathrm{cl} \mathrm{D})$ and $x \in \mathcal{X}$ such that the linear matrix equation

$$
\left[\begin{array}{c}
x^{T} A_{1} \\
\vdots \\
x^{T} A_{k}
\end{array}\right]=c d^{T}
$$

is satisfied. A coordinate-free description can be formulated similarly as in the dual case.

We remark here that the coordinate-free description is handy for the purpose of checking the condition in many applications.

Now we are in a position to state the following extended duality theorem for the D-induced duality. 


\section{Theorem 2.3 Let the bilinear mapping}

$$
\langle x, y\rangle:=\left[\begin{array}{c}
x^{T} A_{1} y \\
\vdots \\
x^{T} A_{k} y
\end{array}\right]:(x, y) \in \mathcal{X} \times \mathcal{Y} \rightarrow \mathcal{W}
$$

be fixed. Let $\mathrm{D} \subseteq \mathcal{W}$ be a given non-flat convex cone. Suppose that $\langle\cdot, \cdot\rangle$ is dual surjective with respect to $\mathrm{D}$. Let $U \subseteq \mathcal{X}$ be a convex cone. Then it holds that

$$
\operatorname{cl} U_{\mathrm{DD}}^{* *}=\operatorname{cl} U
$$

Proof First we prove $\mathrm{cl} U \subseteq \operatorname{cl} U_{\mathrm{DD}}^{* *}$.

Take any $x \in U$. Then by definition, $\langle x, y\rangle \in \mathrm{D}$ for all $y \in U_{\mathrm{D}}^{*}$. Hence, $x \in U_{\mathrm{DD}}^{* *}$, and so it follows that $U \subseteq U_{\mathrm{DD}}^{* *}$. Consequently, it follows that cl $U \subseteq \operatorname{cl} U_{\mathrm{DD}}^{* *}$.

Next we shall prove $U_{\mathrm{DD}}^{* *} \subseteq \mathrm{cl} U$. The following observation will be useful: let $L(y):=\left[A_{1} y, \ldots, A_{k} y\right]$. Then $\langle x, y\rangle=\left(x^{T} L(y)\right)^{T}$, and

$$
\begin{aligned}
y \in U_{\mathrm{cl} \mathrm{D}}^{*} & \Longleftrightarrow\langle u, y\rangle \in \operatorname{cl} \mathrm{D} \text { for all } u \in U \\
& \Longleftrightarrow\left[u^{T} A_{1} y, \ldots, u^{T} A_{k} y\right] v \geq 0 \text { for all } u \in U \text { and } v \in \mathrm{D}^{*} \\
& \Longleftrightarrow u^{T} L(y) v \geq 0 \text { for all } u \in U \text { and } v \in \mathrm{D}^{*} .
\end{aligned}
$$

In other words,

$$
U_{\mathrm{cl} \mathrm{D}}^{*}=\left\{y \mid u^{T} L(y) v \geq 0 \text {, for all } u \in U, v \in \mathrm{D}^{*}\right\} .
$$

Take any $\hat{x} \in U_{\mathrm{DD}}^{* *}$. Thus $\langle\hat{x}, y\rangle \in \mathrm{D}$ for all $y \in U_{\mathrm{D}}^{*}$. Suppose by contradiction that $\hat{x} \notin \mathrm{cl} U$. Then by the separation theorem, there exists $a \in U^{*}$ such that $a^{T} \hat{x}<0$. By the dual surjectivity of the bilinear mapping, we can find $\hat{y}$ such that

$$
L(\hat{y})=a b^{T}
$$

for some non-flat direction $b \in \mathrm{D}$. Hence

$$
u^{T} L(\hat{y}) v=\left(u^{T} a\right)\left(b^{T} v\right) \geq 0
$$

for all $u \in U$ and $v \in \mathrm{D}^{*}$ as $a \in U^{*}$ and $b \in \mathrm{D}$. Consequently, $\langle u, \hat{y}\rangle=L(\hat{y})^{T} u \in$ $\operatorname{clD}$ for all $u \in U$. This shows that $\hat{y} \in U_{\mathrm{cl}}^{*}$. This leads us to the following contradiction. On the one hand, $\langle\hat{x}, \hat{y}\rangle \in \operatorname{cl} \mathrm{D}$ due to the fact that $\hat{x} \in U_{\mathrm{DD}}^{* *}$ and $\hat{y} \in U_{\mathrm{cl} \mathrm{D}}^{*}=\operatorname{cl} U_{\mathrm{D}}^{*}$, where we have used Proposition 2.1. On the other hand,

$$
\langle\hat{x}, \hat{y}\rangle=\left(\hat{x}^{T} a b^{T}\right)^{T}=\left(a^{T} \hat{x}\right) b \notin \mathrm{cl} \mathrm{D},
$$


due to the fact that $a^{T} \hat{x}<0$ and $b$ is a non-flat direction for $\mathrm{D}$. This contradiction proves $U_{\mathrm{DD}}^{* *} \subseteq \mathrm{cl} U$. The desired result follows by taking closure on both sides.

In the same vein, we have an analogue for the dual space.

Theorem 2.4 Let the bilinear mapping

$$
\langle x, y\rangle:=\left[\begin{array}{c}
x^{T} A_{1} y \\
\vdots \\
x^{T} A_{k} y
\end{array}\right]:(x, y) \in \mathcal{X} \times \mathcal{Y} \rightarrow \mathcal{W}
$$

be fixed. Let $\mathrm{D} \subseteq \mathcal{W}$ be a given non-flat convex cone. Suppose that $\langle\cdot, \cdot\rangle$ is primal surjective with respect to $\mathrm{D}$. Let $V \subseteq \mathcal{Y}$ be a convex cone. Then it holds that

$$
\mathrm{cl} V_{\mathrm{DD}}^{* *}=\mathrm{cl} V .
$$

As a matter of notation, let us introduce the tensor product of two convex cones $C \subseteq \Re^{r}$ and $D \subseteq \Re^{s}$ as follows

$$
C \otimes D=\operatorname{conv}\left\{u v^{T} \mid u \in C, v \in D\right\} \subseteq \Re^{r \times s} .
$$

We call its dual the bi-positive cone, denoted by

$$
\mathcal{B}(C, D)=\left\{Z \in \Re^{r \times s} \mid u^{T} Z v \geq 0 \text { for all } u \in C, v \in D\right\} .
$$

Indeed it is elementary to see that

$$
(C \otimes D)^{*}=\mathcal{B}(C, D) .
$$

A proof for the above equality and other related equalities can be found in [6]. In the proof for Theorem 2.3 we established the relation (1). Let us formalize it as follows, now using the notion of the bi-positive cone.

Proposition 2.5 Let $U \subseteq \mathcal{X}$ be a convex cone. Then it holds that

$$
U_{\mathrm{cl} \mathrm{D}}^{*}=\left\{y \mid L(y) \in \mathcal{B}\left(U, \mathrm{D}^{*}\right)\right\} .
$$

Thus the following result is straightforward.

Proposition 2.6 Consider convex cones $U_{1}, \ldots, U_{r} \subseteq \mathcal{X}$. It holds that

$$
\left(U_{1}+\cdots+U_{r}\right)_{\mathrm{cl} \mathrm{D}}^{*}=\bigcap_{i=1}^{r}\left(U_{i}\right)_{\mathrm{cl} \mathrm{D}}^{*}
$$


Similarly, for convex cones $V_{1}, \ldots, V_{r} \subseteq \mathcal{Y}$,

$$
\left(V_{1}+\cdots+V_{r}\right)_{\mathrm{cl} \mathrm{D}}^{*}=\bigcap_{i=1}^{r}\left(V_{i}\right)_{\mathrm{cl} \mathrm{D}}^{*}
$$

Proof We only need to show the first equation. According to Proposition 2.5, we have

$$
\begin{aligned}
\left(U_{1}+\cdots+U_{r}\right)_{\mathrm{cl} \mathrm{D}}^{*} & =\left\{y \mid L(y) \in \mathcal{B}\left(U_{1}+\cdots+U_{r}, \mathrm{D}^{*}\right)\right\} \\
& =\left\{y \mid L(y) \in \bigcap_{i=1}^{r} \mathcal{B}\left(U_{i}, \mathrm{D}^{*}\right)\right\} \\
& =\bigcap_{i=1}^{r}\left(U_{i}\right)_{\mathrm{cl} \mathrm{D}}^{*} .
\end{aligned}
$$

In the reverse direction the following result holds true. Here it has to be kept in mind that, unlike in the usual case, the primal and dual status of the D-induced duality is not symmetric in general. For instance, in cases where the bilinear mapping is dual surjective, it can happen that not all closed convex cones in $\mathcal{Y}$ can be expressed as the dual of some cone in $\mathcal{X}$. However, if the bilinear mapping is both primal and dual surjective, then one can apply the extended duality theorem on both sides of the equality in Proposition 2.6 and this gives the announced converse result.

Corollary 2.7 Suppose that $\langle\cdot, \cdot\rangle$ is both primal and dual surjective with respect to $\mathrm{D}$ and that $\mathrm{D}$ is closed. Let $U_{1}, \ldots, U_{r}$ be arbitrary convex cones in $\mathcal{X}$. Then it holds that

$$
\mathrm{cl}\left(\bigcap_{i=1}^{r} \mathrm{cl} U_{i}\right)_{\mathrm{D}}^{*}=\mathrm{cl}\left(\left(U_{1}\right)_{\mathrm{D}}^{*}+\cdots+\left(U_{r}\right)_{\mathrm{D}}^{*}\right) .
$$

Proof Substitute $V_{i}=\left(U_{i}\right)_{\mathrm{D}}^{*}$ and $\left(V_{i}\right)_{\mathrm{D}}^{*}=\left(\left(U_{i}\right)_{\mathrm{D}}^{*}\right)_{\mathrm{D}}^{*}=\mathrm{cl} U_{i}$ (according to Theorem 2.3) into

$$
\left(V_{1}+\cdots+V_{r}\right)_{\mathrm{D}}^{*}=\bigcap_{i=1}^{r}\left(V_{i}\right)_{\mathrm{D}}^{*},
$$

(Proposition 2.6), we obtain

$$
\left(\left(U_{1}\right)_{\mathrm{D}}^{*}+\cdots+\left(U_{r}\right)_{\mathrm{D}}^{*}\right)_{\mathrm{D}}^{*}=\bigcap_{i=1}^{r} \mathrm{cl} U_{i} .
$$


Taking the D-induced dual and then the closure of the above identity, and then applying Theorem 2.4 to the left-hand-side of the resulting expression, we get

$$
\operatorname{cl}\left(\bigcap_{i=1}^{r} \mathrm{cl} U_{i}\right)_{\mathrm{D}}^{*}=\operatorname{cl}\left(\left(U_{1}\right)_{\mathrm{D}}^{*}+\cdots+\left(U_{r}\right)_{\mathrm{D}}^{*}\right),
$$

completing the proof.

It is in fact quite rare that both the primal and the dual surjectivity conditions are satisfied at the same time. This essentially means that we are dealing with the ordinary duality with the usual inner product and $D=\Re_{+}$.

Other simple calculus rules for the cone-induced duality are presented below.

Proposition 2.8 Let $U$ be a convex cone in $\mathcal{X}$.

(i) For any convex cones $\mathrm{D}_{1}, \ldots, \mathrm{D}_{s}$ in $\mathcal{W}$, it holds that

$$
U_{\mathrm{D}_{1} \cap \cdots \cap \mathrm{D}_{s}}^{*}=U_{\mathrm{D}_{1}}^{*} \cap \cdots \cap U_{\mathrm{D}_{s}}^{*}
$$

and,

(ii)

$$
U_{\mathrm{D}_{1}+\cdots+\mathrm{D}_{s}}^{*} \supseteq U_{\mathrm{D}_{1}}^{*}+\cdots+U_{\mathrm{D}_{s}}^{*} .
$$

(iii) Suppose that the bilinear mapping is decomposed as

$$
\langle x, y\rangle=\left[\begin{array}{c}
\langle x, y\rangle_{1} \\
\vdots \\
\langle x, y\rangle_{p}
\end{array}\right]
$$

where $\langle x, y\rangle_{i}$ is a bilinear mapping from $\mathcal{X} \times \mathcal{Y}$ to $\mathcal{W}_{k_{i}}$ where $\mathcal{W}_{k_{i}}=\Re^{k_{i}}$ with $\sum_{i=1}^{p} k_{i}=k$. Furthermore, suppose that convex cones $\mathrm{D}_{i} \subseteq \mathcal{W}_{k_{i}}$ are given, $i=1, \ldots, p$. Then it holds that

$$
U_{\mathrm{D}_{1} \times \cdots \times \mathrm{D}_{p}}^{*}=U_{\mathrm{D}_{1}}^{*} \cap \cdots \cap U_{\mathrm{D}_{p}}^{*}
$$

where the set product is defined as

$$
\mathrm{D}_{1} \times \cdots \times \mathrm{D}_{p}=\left\{\left(z_{1}, \ldots, z_{p}\right) \mid z_{i} \in \mathrm{D}_{i}, i=1, \ldots, p\right\} .
$$

Proof To show (i) we note that

$$
\begin{aligned}
y \in U_{\mathrm{D}_{1} \cap \cdots \cap \mathrm{D}_{s}}^{*} & \Longleftrightarrow\langle U, y\rangle \in \mathrm{D}_{i}, i=1, \ldots, s \\
& \Longleftrightarrow y \in U_{\mathrm{D}_{i}}^{*}, i=1, \ldots, s \\
& \Longleftrightarrow y \in U_{\mathrm{D}_{1}}^{*} \cap \cdots \cap U_{\mathrm{D}_{s}}^{*} .
\end{aligned}
$$


For proving (ii) we note that if $y \in U_{\mathrm{D}_{1}}^{*}+\cdots+U_{\mathrm{D}_{s}}^{*}$ then there exist $y_{1}, \ldots, y_{s}$ such that $y=y_{1}+\cdots+y_{s}$, and $y_{i} \in U_{\mathrm{D}_{i}}^{*}, i=1, \ldots, s$. This implies that $\left\langle U, y_{i}\right\rangle \subseteq \mathrm{D}_{i}, i=1, \ldots, s$, and so

$$
\langle U, y\rangle \subseteq\left\langle U, y_{1}\right\rangle+\cdots+\left\langle U, y_{s}\right\rangle \subseteq \mathrm{D}_{1}+\cdots+\mathrm{D}_{s} .
$$

Thus, $U_{\mathrm{D}_{1}+\cdots+\mathrm{D}_{s}}^{*} \supseteq U_{\mathrm{D}_{1}}^{*}+\cdots+U_{\mathrm{D}_{s}}^{*}$.

Note that the inclusion above is strict in general. For more discussions on this, see Section 6.

Now we prove (iii). Similarly as in (i),

$$
\begin{aligned}
y \in U_{\mathrm{D}_{1} \times \cdots \times \mathrm{D}_{p}}^{*} & \Longleftrightarrow\langle U, y\rangle_{i} \in \mathrm{D}_{i}, i=1, \ldots, p \\
& \Longleftrightarrow y \in U_{\mathrm{D}_{i}}^{*}, i=1, \ldots, p \\
& \Longleftrightarrow y \in U_{\mathrm{D}_{1}}^{*} \cap \cdots \cap U_{\mathrm{D}_{p}}^{*} .
\end{aligned}
$$

Remark here that the statements in (i) and (iii) are different: in (i), the $\mathrm{D}_{i}$ 's are all contained in $\Re^{k}$, while in (iii), $\mathrm{D}_{i}$ is in $\Re^{k_{i}}, i=1, \ldots, p$.

By (iii) of Proposition 2.8 it is clear that one needs only to concentrate on the case that the underlying cone $D$ is non-decomposable, for the duality consideration.

Let us see how the D-induced duality actually works.

\section{Example Consider}

$$
\mathcal{X}=\mathcal{S}^{n}, \mathcal{Y}=\left\{\left[\begin{array}{ll}
Y_{11} & Y_{12} \\
Y_{12} & Y_{22}
\end{array}\right] \in \mathcal{S}^{2 n} \mid Y_{12} \in \mathcal{S}^{n}\right\}, \quad \mathcal{W}=\mathcal{S}^{2} .
$$

The bilinear mapping is

$$
\langle X, Y\rangle=\left[\begin{array}{ll}
X \bullet Y_{11} & X \bullet Y_{12} \\
X \bullet Y_{12} & X \bullet Y_{22}
\end{array}\right]
$$

and $\mathrm{D}=\mathcal{S}_{+}^{2}$, where $\bullet$ denotes the usual entry-wise inner product for matrices.

In this case, the bilinear mapping is dual surjective. To see this, let us fix a non-flat direction of $\mathrm{D}$, e.g. $B=\left[\begin{array}{ll}1 & 1 \\ 1 & 1\end{array}\right]$. Then, for any $A \in \mathcal{X}$ the choice $Y=\left[\begin{array}{ll}A & A \\ A & A\end{array}\right]$ satisfies the dual surjective requirement: $\langle X, Y\rangle=(X \bullet A) B$ for all $X \in \mathcal{X}$.

By Theorem 4.5 of Luo, Sturm and Zhang [6] we have

$$
\left(\mathcal{S}_{+}^{n}\right)_{\mathcal{S}_{+}^{2}}^{*}=\left\{\left[\begin{array}{ll}
Z_{11} & Z_{12} \\
Z_{12} & Z_{22}
\end{array}\right] \succeq 0 \mid Z_{12} \in \mathcal{S}^{n}\right\}
$$


The extended duality theorem, Theorem 2.3, thus asserts that

$$
\left\{\left[\begin{array}{ll}
Z_{11} & Z_{12} \\
Z_{12} & Z_{22}
\end{array}\right] \succeq 0 \mid Z_{12} \in \mathcal{S}^{n}\right\}_{\mathcal{S}_{+}^{2}}^{*}=\mathcal{S}_{+}^{n} .
$$

\section{The D-induced polar set}

It is useful to extend the notion of D-induced dual cone to the more general notion D-induced polar set. Following the ordinary duality theory, the polar operation can be considered as a truncation operation after the homogenization and the dual cone operations; the exact meaning of this statement will become clear later. However, in our D-induced duality case, the D-induced polar is flexible enough to simply include the $\mathrm{D}$-induced dual cone as a special case. Certainly, we may also view the D-induced polar set as a truncation of the D-induced dual cone in a homogenized space. Hence, in this respect, these two notions are equivalent. For practical purposes however, the D-induced polar set is important in its own right.

Before we discuss the $\mathrm{D}$-induced polar, let us briefly introduce the usual polar duality theory for the sake of making comparisons. For details of duality theory, use Rockafellar [11] as a standard reference.

Let $S \in \mathcal{X}$ be a convex set. Its polar is a closed convex set defined as $S^{o}=\left\{y \mid\langle x, y\rangle_{\mathcal{X}}+1 \geq 0\right.$ for all $\left.x \in S\right\}$. The standard bi-polar theorem in this context states: if $0 \in S$ then $\left(S^{o}\right)^{o}=\operatorname{cl} S$. It is also well known that the ordinary conic form bi-polar theorem, i.e. Theorem 2.4 with $\langle x, y\rangle=\langle x, y\rangle \mathcal{X}$ and $\mathrm{D}=\Re_{+}$, leads to the statement above. To see this, consider first the following homogenization operation. Let $\overline{\mathcal{X}}:=\Re \times \mathcal{X}$. For a given set $S \in \mathcal{X}$ define

$$
H(S):=\operatorname{cl}\left\{\left[\begin{array}{c}
x_{0} \\
x
\end{array}\right] \mid x_{0}>0, x / x_{0} \in S\right\} \subseteq \overline{\mathcal{X}} .
$$

Let us also define the following anti-polar set:

$$
S^{a}=\left\{y \mid\langle x, y\rangle_{\mathcal{X}}+1 \leq 0 \text { for all } x \in S\right\} .
$$

Obviously, if $0 \in S$ then $S^{a}=\emptyset$.

We now prove a general relationship between the dual cone and the polar/ anti-polar sets.

Theorem 3.1 It holds that $H(S)^{*}=\mathrm{cl}\left(H\left(S^{o}\right)-H\left(S^{a}\right)\right)$.

Proof Take any $\left[y_{0}, y^{T}\right]^{T} \in\left(H\left(S^{o}\right)-H\left(S^{a}\right)\right)$ with

$$
\left[y_{0}, y^{T}\right]^{T}=\left[y_{0}^{\prime}, y^{\prime T}\right]^{T}-\left[y_{0}^{\prime \prime}, y^{\prime \prime T}\right]^{T},
$$


where $y_{0}^{\prime}>0, y_{0}^{\prime \prime}>0, y^{\prime} / y_{0}^{\prime} \in S^{o}$ and $y^{\prime \prime} / y_{0}^{\prime \prime} \in S^{a}$. Then, for any $\left[x_{0}, x^{T}\right]^{T}$ with $x_{0}>0$ and $x / x_{0} \in S$, we have

$$
\begin{aligned}
x_{0} y_{0}+x^{T} y & =x_{0}\left(y_{0}^{\prime}-y_{0}^{\prime \prime}\right)+x^{T}\left(y^{\prime}-y^{\prime \prime}\right) \\
& =x_{0} y_{0}^{\prime}\left[\left(\frac{x}{x_{0}}\right)^{T}\left(\frac{y^{\prime}}{y_{0}^{\prime}}\right)+1\right]-x_{0} y_{0}^{\prime \prime}\left[\left(\frac{x}{x_{0}}\right)^{T}\left(\frac{y^{\prime \prime}}{y_{0}^{\prime \prime}}\right)+1\right] \\
& \geq 0
\end{aligned}
$$

leading to the fact that $\left[y_{0}, y^{T}\right]^{T} \in H(S)^{*}$. Taking the closure we have cl $\left(H\left(S^{o}\right)\right.$ $\left.-H\left(S^{a}\right)\right) \subseteq H(S)^{*}$.

Next we consider the other containment relation.

Take any $\left[y_{0}, y^{T}\right]^{T} \in H(S)^{*}$. We have $x_{0} y_{0}+x^{T} y \geq 0$ for all $x_{0}>0$ and $x / x_{0} \in S$, in particular, $y_{0}+x^{T} y \geq 0$ for all $x \in S$. If $y_{0}>0$ then $x^{T}\left(y / y_{0}\right)+1 \geq 0$ for all $x \in S$, thus $\left[y_{0}, y^{T}\right]^{T} \in H\left(S^{o}\right)$. If $y_{0}=0$ then we may take an arbitrary $\epsilon>0$ and conclude that $\epsilon+x^{T} y \geq x^{T} y \geq 0$ for all $x \in S$. Therefore, $\left[\epsilon, y^{T}\right]^{T} \in H\left(S^{o}\right)$. Letting $\epsilon \downarrow 0$ yields $\left[y_{0}, y^{T}\right]^{T}=\left[0, y^{T}\right]^{T} \in \operatorname{cl} H\left(S^{o}\right)$. If $y_{0}<0$ then $x^{T}\left(\frac{-y}{-y_{0}}\right)+1 \leq 0$ for all $x \in S$, implying that $-\left[y_{0}, y^{T}\right]^{T} \in H\left(S^{a}\right)$. Therefore, $H(S)^{*} \subseteq \operatorname{cl}\left(H\left(S^{o}\right)-H\left(S^{a}\right)\right)$.

Corollary 3.2 If $0 \in S$ then $H(S)^{*}=H\left(S^{o}\right)$ and $S^{o o}=\operatorname{cl} S$.

Proof If $0 \in S$ then $S^{a}=\emptyset$ and so by Theorem 3.1 we have $H(S)^{*}=H\left(S^{o}\right)$. Now we use the standard bi-polar theorem for convex cones to obtain

$$
H(S)=H(S)^{* *}=\left(H(S)^{*}\right)^{*}=\left(H\left(S^{o}\right)\right)^{*}=H\left(S^{o o}\right) .
$$

Therefore, $S^{o o}=\operatorname{cl} S$.

Next we proceed to consider the D-induced polar sets. As before, let $\langle x, y\rangle$ : $(x, y) \in \mathcal{X} \times \mathcal{Y} \rightarrow \mathcal{W}$ be the bilinear mapping under consideration, and using a suitable coordinate system we may assume

$$
\langle x, y\rangle=\left[\begin{array}{c}
x^{T} A_{1} y \\
\vdots \\
x^{T} A_{k} y
\end{array}\right] .
$$

Let $\mathrm{D}$ be a given convex set in $\mathcal{W}$. For simplicity, in this section we assume $\mathrm{D}$ to be a closed set. Let $S \subseteq \mathcal{X}$. We call

$$
S_{\mathrm{D}}^{o}=\{y \in \mathcal{Y} \mid\langle x, y\rangle \in \mathrm{D} \text { for all } x \in S\}
$$

the D-induced polar of $S$. Note that the ordinary polar corresponds to $\mathrm{D}=$ $[-1, \infty)$, that the anti-polar corresponds to $(-\infty,-1]$, and that if $S$ is a cone and $D=\Re_{+}$then we get the traditional dual. Clearly, the D-induced polar set is convex. However it can be empty if no additional assumption is imposed. In most of our analysis, we assume $0 \in \mathrm{D}$, and so $0 \in S_{\mathrm{D}}^{o} \neq \emptyset$. In case $\mathrm{D}$ itself is a 
convex cone, the D-induced polar set of a cone $U$ is simply its $\mathrm{D}$-induced dual cone. In this sense, unlike the ordinary polarity, the D-induced polar includes the D-induced dual cone as a special case. We shall see below that these notions are actually equivalent, under the homogenization operation. It is evident that the calculus rules as established in Proposition 2.8 are valid in the original form for the D-induced polar sets as well. However, Proposition 2.6 needs a slight modification to accommodate the fact that convex sets are under consideration, rather than convex cones.

Proposition 3.3 Let $S_{i} \subseteq \mathcal{X}$ be convex sets, $i=1, \ldots, r$. Then,

$$
\left(\operatorname{conv}\left(\bigcup_{i=1}^{r} S_{i}\right)\right)_{\mathrm{D}}^{o}=\bigcap_{i=1}^{r}\left(S_{i}\right)_{\mathrm{D}}^{o} .
$$

Furthermore, if $\mathrm{D}$ is a convex cone, then

$$
\left(\sum_{i=1}^{r} S_{i}\right)_{\mathrm{D}}^{o}=\bigcap_{i=1}^{r}\left(S_{i}\right)_{\mathrm{D}}^{o} \cdot
$$

Proof Clearly, $x \in\left(\operatorname{conv}\left(\bigcup_{i=1}^{r} S_{i}\right)\right)_{\mathrm{D}}^{o}$ if and only if $\sum_{i=1}^{r}\left\langle x, \lambda_{i} y_{i}\right\rangle \in \mathrm{D}$ for all $y_{i} \in S_{i}$, and $\lambda_{i} \geq 0$ and $\sum_{i=1}^{r} \lambda_{i}=1$. The latter is equivalent to $\left\langle x, y_{i}\right\rangle \in \mathrm{D}$ for all $y_{i} \in S_{i}, i=1, \ldots, r$; in other words, $x \in \bigcap_{i=1}^{r}\left(S_{i}\right)_{\mathrm{D}}^{o}$. In case $\mathrm{D}$ is a cone, then $\left(\text { conv }\left(\bigcup_{i=1}^{r} S_{i}\right)\right)_{\mathrm{D}}^{o}=\left(\sum_{i=1}^{r} S_{i}\right)_{\mathrm{D}}^{o}$, and so the second statement follows from the first one.

Let $\overline{\mathcal{Y}}:=\Re \times \mathcal{Y}$ and $\overline{\mathcal{W}}:=\Re \times \mathcal{W}$. For $\bar{x}=\left[x_{0}, x^{T}\right]^{T} \in \overline{\mathcal{X}}$ and $\bar{y}=\left[y_{0}, y^{T}\right]^{T} \in$ $\overline{\mathcal{Y}}$, consider the following extended bilinear mapping

$$
\langle\bar{x}, \bar{y}\rangle:=\left[\begin{array}{l}
x_{0} y_{0} \\
\langle x, y\rangle
\end{array}\right] \text {. }
$$

The order-defining cone is naturally chosen to be $H(\mathrm{D})$. The first result is immediate.

Proposition 3.4 For any $S \subseteq \mathcal{X}$ with $0 \in S$, we have $H(S)_{H(\mathrm{D})}^{*}=H\left(S_{\mathrm{D}}^{o}\right)$.

Proof Take any $\left[y_{0}, y^{T}\right]^{T} \in H(S)_{H(\mathrm{D})}^{*}$. We have

$$
\left[\begin{array}{c}
x_{0} y_{0} \\
\langle x, y\rangle
\end{array}\right] \in H(\mathrm{D}) \text { for all }\left[x_{0}, x^{T}\right]^{T} \in H(S) \text {. }
$$

Hence, $y_{0} \geq 0$. Consider first $y_{0}>0$. In that case, $\left\langle x, \frac{y}{y_{0}}\right\rangle \in \mathrm{D}$ for all $x \in S$, and so $\left[y_{0}, y^{T}\right]^{T} \in H\left(S_{\mathrm{D}}^{o}\right)$. If $y_{0}=0$ then $\left[0, y^{T}\right]^{T} \in H(S)_{H(\mathrm{D})}^{*}$ meaning that there is a sequence $\epsilon_{n} \downarrow 0$ and $y_{n} \rightarrow y$ such that $\left\langle x, y_{n} / \epsilon_{n}\right\rangle \in \mathrm{D}$ for all $x \in S$. This implies that $\left[\epsilon_{n}, y_{n}^{T}\right]^{T} \in H\left(S_{\mathrm{D}}^{o}\right)$. Taking limit yields $\left[y_{0}, y^{T}\right]^{T} \in H\left(S_{\mathrm{D}}^{o}\right)$. 
Next we show the converse containment relationship. Take $\left[y_{0}, y^{T}\right]^{T} \in H\left(S_{\mathrm{D}}^{o}\right)$ with $y_{0}>0$. We thus have $\left\langle x, \frac{y}{y_{0}}\right\rangle \in \mathrm{D}$ for all $x \in S$. Now take any $\left[x_{0}, x^{T}\right]^{T} \in$ $H(S)$ with $x_{0}>0$. We have $\left\langle\frac{x}{x_{0}}, \frac{y}{y_{0}}\right\rangle \in \mathrm{D}$, or equivalently,

$$
\left[\begin{array}{c}
x_{0} y_{0} \\
\langle x, y\rangle
\end{array}\right] \in H(\mathrm{D}) \text {. }
$$

Taking the closure it follows that $H\left(S_{\mathrm{D}}^{o}\right) \subseteq H(S)_{H(\mathrm{D})}^{*}$. The proposition is proven.

For the extended D-induced bi-polar theorem, we introduce the following corresponding condition on surjectivity.

Definition 3.5 We call $\langle x, y\rangle$ dual surjective with respect to the set $\mathrm{D}$ if for any $a \in \mathcal{X}$ there is $b \in \mathrm{D}$ with $-\lambda b \notin \mathrm{D}$ for all $\lambda>1$, and an element $y \in \mathcal{Y}$ such that the linear equation

$$
\left[A_{1} y, \ldots, A_{k} y\right]=a b^{T}
$$

is satisfied. The coordinate-free description is

$\forall a \in \mathcal{X}, \exists b \in \mathrm{D}(-\lambda b \in \mathrm{D} \forall \lambda>1), \exists y \in Y$, such that $\langle x, y\rangle=\langle x, a\rangle \mathcal{X} b$ for all $x \in \mathcal{X}$.

The extended duality theorem for the D-induced duality (Theorem 2.3) can be generalized as follows.

Theorem 3.6 Suppose that the bilinear mapping $\langle x, y\rangle$ is dual surjective according to Definition 3.5. Moreover, suppose that $\mathrm{D}$ is symmetric around the origin. Then for any convex set $S \subseteq \mathcal{X}$ which is symmetric around the origin, we have

$$
\left((H(S))_{H(\mathrm{D})}^{*}\right)_{H(\mathrm{D})}^{*}=H(S)
$$

Proof That $H(S) \subseteq\left((H(S))_{H(\mathrm{D})}^{*}\right)_{H(\mathrm{D})}^{*}$ is obvious. We shall concentrate on proving the converse containment relationship.

We shall first identify the set $(H(S))_{H(\mathrm{D})}^{*}$. Let $\bar{y}=\left[y_{0}, y^{T}\right]^{T} \in(H(S))_{H(\mathrm{D})}^{*}$. This requires that

$$
\left[\begin{array}{l}
x_{0} y_{0} \\
\langle x, y\rangle
\end{array}\right] \in H(\mathrm{D}) \text { for all }\left[x_{0}, x^{T}\right]^{T} \in H(S) \text {. }
$$

Or, equivalently,

$$
x_{0} y_{0} w_{0}+x^{T} L(y) w \geq 0
$$

for all $\left[w_{0}, w^{T}\right]^{T} \in H(\mathrm{D})^{*}=H\left(\mathrm{D}^{o}\right)\left(\right.$ Corollary 3.2), and $\left[x_{0}, x^{T}\right]^{T} \in H(S)$, where $L$ is again the linear mapping $Y \rightarrow \Re^{n \times k}$ for which $\langle x, y\rangle=\left(x^{T} L(y)\right)^{T}$ for all $x \in \mathcal{X}, y \in \mathcal{Y}$. 
Suppose by contradiction that there is

$$
\left[\begin{array}{c}
\hat{x}_{0} \\
\hat{x}
\end{array}\right] \in\left((H(S))_{H(\mathrm{D})}^{*}\right)_{H(\mathrm{D})}^{*} \backslash H(S) .
$$

By the separation theorem, there is $\left[a_{0}, a^{T}\right]^{T} \in H(S)^{*}=H\left(S^{o}\right)$ such that

$$
a_{0} \hat{x}_{0}+a^{T} \hat{x}<0
$$

Using the surjectivity condition, there is $\hat{y}$ such that $L(\hat{y})=a b^{T}$ with $b \in \mathrm{D}$ and $-\lambda b \notin \mathrm{D}$ for all $\lambda>1$. Now we claim that $\bar{y}=\left[a_{0}, \hat{y}^{T}\right]^{T}$ satisfies (7). To see this we note that

$$
\begin{aligned}
& a_{0} x_{0}+a^{T} x \geq 0, \forall\left[x_{0}, x^{T}\right]^{T} \in H(S) \quad\left(\text { since }\left[a_{0}, a^{T}\right]^{T} \in H(S)^{*}\right), \\
& \left.w_{0}+w^{T} b \geq 0, \forall\left[w_{0}, w^{T}\right]^{T} \in H(\mathrm{D})^{*} \quad \text { (since }\left[1, b^{T}\right]^{T} \in H(\mathrm{D})\right), \\
& a_{0} \geq 0, x_{0} \geq 0, w_{0} \geq 0 \quad \text { (due to the homogenization operation). }
\end{aligned}
$$

In fact, since $S$ and D are symmetric around the origin, we know that $S^{o}$ and $\mathrm{D}^{o}$ are also symmetric around the origin. Therefore, inequalities (9) and (10) further imply that

$$
a_{0} x_{0}-\left|a^{T} x\right| \geq 0, \quad \text { for all }\left[x_{0}, x^{T}\right]^{T} \in H(S),
$$

and

$$
w_{0}-\left|w^{T} b\right| \geq 0, \quad \text { for all }\left[w_{0}, w^{T}\right]^{T} \in H(\mathrm{D})^{*} .
$$

Using (11), (12) and (13) we have

$$
a_{0} x_{0} w_{0}+x^{T} L(\hat{y}) w=a_{0} x_{0} w_{0}+a^{T} x b^{T} w \geq 0
$$

for all $\left[w_{0}, w^{T}\right]^{T} \in H(\mathrm{D})^{*}$ and $\left[x_{0}, x^{T}\right]^{T} \in H(S)$. Hence $\left[a_{0}, \hat{y}^{T}\right]^{T} \in(H(S))_{H(\mathrm{D})}^{*}$.

Moreover,

$$
\left\langle\left[\begin{array}{c}
\hat{x}_{0} \\
\hat{x}
\end{array}\right],\left[\begin{array}{c}
a_{0} \\
\hat{y}
\end{array}\right]\right\rangle=\left[\begin{array}{c}
a_{0} \hat{x}_{0} \\
\langle\hat{x}, \hat{y}\rangle
\end{array}\right]=\left[\begin{array}{c}
a_{0} \hat{x}_{0} \\
\left(a^{T} \hat{x}\right) b
\end{array}\right]
$$

where for the last equality we use $\langle\hat{x}, \hat{y}\rangle=\left(\hat{x}^{T} L(\hat{y})\right)^{T}=\left(\hat{x}^{T} a b^{T}\right)^{T}=\left(a^{T} \hat{x}\right) b$. By (8) and the non-flatness of $b$ it follows that $\left[a_{0}, \hat{y}^{T}\right]^{T} \notin(H(S))_{H(\mathrm{D})}^{*}$. Thus we have established both inclusion and non inclusion, yielding a contradiction. This establishes the required converse containment relation. Therefore it follows that

$$
\left((H(S))_{H(\mathrm{D})}^{*}\right)_{H(\mathrm{D})}^{*}=H(S)
$$

Using Proposition 3.4 we further obtain: 
Corollary 3.7 Suppose that the bilinear mapping $\langle x, y\rangle$ is dual surjective according to Definition 3.5. Moreover, suppose that $\mathrm{D}$ is symmetric around the origin. Then for any convex set $S \subseteq \mathcal{X}$ which is symmetric around the origin, we have $\left(S_{\mathrm{D}}^{o}\right)_{\mathrm{D}}^{o}=\mathrm{cl} S$.

\section{Computing the D-induced dual objects}

Undoubtedly, theoretical properties such as the bipolar theorems are important for convex analysis. For practical purposes, however, it is also important to be able to compute the dual objects in a tangible way. This will be made precise below using the concept LMI (Linear Matrix Inequalities). To begin with we consider the problem of computing an ordinary dual object. It is well known that this computation is essentially of the same complexity as the computation of the primal object itself. In particular, it is shown by Grötschel et al. [4] (Theorem 4.4.4) that if there is an oracle polynomial-time procedure to check the weak membership for the original convex set, then there is also an oracle polynomial-time procedure to check the weak membership for its polar set.

The situation becomes quite blurred in the D-induced duality. It may happen that both $S$ and D are simple convex sets, but the membership check for $S_{\mathrm{D}}^{o}$ becomes a hard task. Specifically we consider the following result due to Nemirovskii and Ben-Tal; see [1]:

Proposition 4.1 For given symmetric matrices $A_{i} \in \mathcal{S}^{m}$, the following decision problem is co-NP complete:

$$
\sum_{i=1}^{n} x_{i} A_{i} \preceq I, \quad \text { for all } x \in \Re^{n} \text { with }\|x\| \leq 1 .
$$

In the terminology of D-induced duality, this implies the following. Let us consider $\mathcal{X}=\Re^{n}, \mathcal{Y}=\left(\mathcal{S}^{m}\right)^{n}$ (the Cartesian product of $n$ copies of the space of $m \times m$ symmetric matrices), and $\mathcal{W}=\mathcal{S}^{m}$. For $x \in \mathcal{X}$ and $y=\left(Y_{1}, \ldots, Y_{n}\right) \in \mathcal{Y}$, let the bilinear product be defined as

$$
\langle x, y\rangle=\sum_{i=1}^{n} x_{i} Y_{i} .
$$

Let $\mathrm{D}=\{W \mid W \preceq I\}$. Then Proposition 4.1 asserts that it is NP-hard to check the membership for the polar set $S_{\mathrm{D}}^{o}$ where $S$ is a Euclidean unit ball in $\mathcal{X}$. Of course, one may homogenize and then reformulate the problem as checking the membership of a $\overline{\mathbf{D}}$-induced dual of a second order cone where $\overline{\mathrm{D}}$ is the $m \times m$ positive semidefinite matrix cone.

However, there are interesting special cases for which the computation can be done satisfactorily. Now let us be more specific about our terminology. As a convention, we shall call the description of a set satisfactory if the description is done by a polynomial number of polynomially-sized Linear Matrix Inequalities 
(LMI), where the polynomials are in terms of the input size of the problem. In short, we call such a description LMI representable. As is well-known, the usual linear inequality representation is the special case of the linear matrix inequality representation where all the matrices are diagonal.

Let us first consider the polyhedral case. Note the famous Farkas lemma (the inhomogeneous version): the implication " $F x \leq f$ (assuming it is nonempty) $\Longrightarrow g^{T} x \leq h$ " holds if and only if $\exists z \geq 0$ and $z_{0} \geq 0$ such that

$$
\left[h, g^{T}\right]=\left[z, z_{0}\right]\left[\begin{array}{ll}
f & F \\
1 & 0
\end{array}\right] \text {. }
$$

Hence, " $F x \leq f$ (assuming it is nonempty) $\Longrightarrow G x \leq h$ " holds if and only if $\exists Z \geq 0$ such that

$$
[h, G]=Z\left[\begin{array}{ll}
f & F \\
1 & 0
\end{array}\right]
$$

Let $\mathcal{X}=\mathfrak{R}^{n}, \mathcal{Y}=\mathfrak{R}^{m}, \mathcal{W}=\mathfrak{R}^{k}$. The bilinear mapping is given as

$$
\langle x, y\rangle=\left[\begin{array}{c}
x^{T} A_{1} y \\
\vdots \\
x^{T} A_{k} y
\end{array}\right] .
$$

Let $\mathrm{D}=\{w \mid D w \leq d\}$ and $S=\{x \mid F x \leq f\}$. Applying the Farkas lemma one obtains

$$
(S)_{\mathrm{D}}^{o}=\left\{y \mid \exists Z \geq 0:\left[d, D L(y)^{T}\right]=Z\left[\begin{array}{ll}
f & F \\
1 & 0
\end{array}\right]\right\},
$$

where $L(y)=\left[A_{1} y, A_{2} y, \ldots, A_{k} y\right]$.

Consider $S=\left\{x \mid x^{T} Q x+2 q^{T} x+q_{0} \leq 0\right\} \subseteq \mathcal{X}$ and $\mathrm{D}=\{w \mid D w \leq d\}$. This is a degenerate case of our next discussion. Let us write $D=\left[g_{1}, g_{2}, \ldots, g_{r}\right]^{T}$ and $d=\left[h_{1}, h_{2}, \ldots, h_{r}\right]^{T}$. Using formula (16) to come, where the general case is discussed, we have

$$
(S)_{\mathrm{D}}^{o}=\left\{y \mid \exists s_{i} \geq 0:\left[\begin{array}{cc}
s_{i} q_{0}+h_{i} & s_{i} q^{T}-\left(L(y) g_{i}\right)^{T} / 2 \\
s_{i} q-L(y) g_{i} / 2 & s_{i} Q
\end{array}\right] \succeq 0, i=1, \ldots, r\right\} .
$$

If $S$ is a polyhedron and D is an ellipsoid, then checking the membership of $(S)_{\mathrm{D}}^{o}$ becomes checking whether a polyhedron is contained in an ellipsoid, which is an NP-hard problem.

We now consider the case where both $S$ and D are level sets of quadratic functions.

Let $\mathrm{D}=\left\{w \mid w^{T} B w+2 b^{T} w+b_{0} \leq 0\right\} \subseteq \mathcal{W}$ with $B=\left[b_{i j}\right]_{k \times k} \in \mathcal{S}_{++}^{k}$ and $b=\left[b_{i}\right]_{k \times 1} \in \mathfrak{R}^{k}$. Consider $S=\left\{x \mid x^{T} Q x+2 q^{T} x+q_{0} \leq 0\right\} \subseteq \mathcal{X}$. Note that $Q$ may be indefinite. For simplicity we assume that int $S \neq \emptyset$. 
It is obvious that in this case $y \in S_{\mathrm{D}}^{o}$ if and only if the following implication holds

$$
\sum_{i=1}^{k} \sum_{j=1}^{k}\left(x^{T} A_{i} y\right)\left(x^{T} A_{j} y\right) b_{i j}+2 \sum_{i=1}^{k} x^{T} A_{i} y b_{i}+b_{0} \leq 0
$$

for all $x$ satisfying

$$
x^{T} Q x+2 q^{T} x+q_{0} \leq 0 .
$$

By the so-called S-Lemma (for the format that suits this context, cf. Theorem 1 in Sturm and Zhang [12]), the above implication is true if and only if there is $s \geq 0$ such that

$$
s\left[\begin{array}{cc}
q_{0} & q^{T} \\
q & Q
\end{array}\right]-\left[\begin{array}{cc}
b_{0} & \left(\sum_{i=1}^{k} b_{i} A_{i} y\right)^{T} \\
\left(\sum_{i=1}^{k} b_{i} A_{i} y\right) & \sum_{i=1}^{k} \sum_{j=1}^{k}\left(A_{i} y y^{T} A_{j}\right) b_{i j}
\end{array}\right] \succeq 0 .
$$

Noting $\left[A_{1} y, A_{2} y, \ldots, A_{k} y\right]=L(y)$ we may write (15) as

$$
s\left[\begin{array}{cc}
q_{0} & q^{T} \\
q & Q
\end{array}\right]-\left[\begin{array}{cc}
b_{0} & (L(y) b)^{T} \\
L(y) b & L(y) B L(y)^{T}
\end{array}\right] \succeq 0,
$$

or,

$$
\left[\begin{array}{cc}
s q_{0}-b_{0} & s q^{T}-(L(y) b)^{T} \\
s q-L(y) b & s Q
\end{array}\right]-\left[\begin{array}{c}
0 \\
L(y)
\end{array}\right] B\left[0, L(y)^{T}\right] \succeq 0 .
$$

Using the Schur complement lemma, (16) can be equivalently written as

$$
\left[\begin{array}{cc}
s q_{0}-b_{0} & s q^{T}-(L(y) b)^{T} \\
s q-L(y) b & s Q
\end{array}\right],\left[\begin{array}{c}
0 \\
L(y) \\
{\left[0, L(y)^{T}\right],}
\end{array}\right] \succeq 0 .
$$

Hence we have the following LMI representation result.

Theorem 4.2 Suppose that $S=\left\{x \mid x^{T} Q x+2 q^{T} x+q_{0} \leq 0\right\} \subseteq \mathcal{X}$ and $\mathrm{D}=\{w \mid$ $\left.w^{T} B w+2 b^{T} w+b_{0} \leq 0\right\} \subseteq \mathcal{W}$ with $B \succ 0$, and the bilinear mapping given as (14). Then it holds that

$$
(S)_{\mathrm{D}}^{o}=\left\{y \mid \exists s \geq 0:\left[\begin{array}{cc}
s q_{0}-b_{0} & s q^{T}-(L(y) b)^{T} \\
s q-L(y) b & s Q \\
{\left[0, L(y)^{T}\right],} & {\left[\begin{array}{c}
0 \\
L(y)
\end{array}\right]} \\
B^{-1}
\end{array}\right] \geq 0\right\} .
$$


By Proposition 3.4 we have $(H(S))_{H(\mathrm{D})}^{o}=H\left(S_{\mathrm{D}}^{o}\right)$, provided that the bilinear mapping is given as (6). Hence, by Theorem 4.2, for $S=\left\{x \mid x^{T} Q x+2 q^{T} x+q_{0}\right.$ $\leq 0\} \subseteq \mathcal{X}$ and $\mathrm{D}=\left\{w \mid w^{T} B w+2 b^{T} w+b_{0} \leq 0\right\} \subseteq \mathcal{W}$,

$$
\begin{aligned}
& (H(S))_{H(\mathrm{D})}^{o} \\
& \left.\quad=\left\{\left[\begin{array}{c}
y_{0} \\
y
\end{array}\right] \mid y_{0} \geq 0, s \geq 0:\left[\begin{array}{cc}
s q_{0}-b_{0} & s q^{T}-(L(y) b)^{T} \\
s q-L(y) b & s Q
\end{array}\right],\left[\begin{array}{c}
0 \\
L(y)
\end{array}\right]\right] \succeq 0\right\} . \\
& {\left[0, L(y)^{T}\right],}
\end{aligned}
$$

Suppose that $B \succeq 0$ is diagonal. Let $\tilde{L}(y):=\left[\tilde{L}_{1}(y), \ldots, \tilde{L}_{k}(y)\right]$ where $\tilde{L}_{i}(y)$ $:=A_{i} y$ if $B_{i i}>0$ and $\tilde{L}_{i}(y)$ is the zero vector if $B_{i i}=0, i=1, \ldots, k$. Then it is straightforward to obtain from Theorem 4.2 the following result, using the pseudo-inverse.

Corollary 4.3 Suppose that $S=\left\{x \mid x^{T} Q x+2 q^{T} x+q_{0} \leq 0\right\} \subseteq \mathcal{X}$ and $\mathrm{D}$ $=\left\{w \mid w^{T} B w+2 b^{T} w+b_{0} \leq 0\right\} \subseteq \mathcal{W}$ with $B=\operatorname{diag}\left(B_{11}, \ldots, B_{k k}\right) \succeq 0$, and the bilinear mapping given as (14). Then it holds that

$$
(S)_{\mathrm{D}}^{o}=\left\{y \mid \exists s \geq 0:\left[\begin{array}{cc}
{\left[\begin{array}{cc}
s q_{0}-b_{0} & s q^{T}-(L(y) b)^{T} \\
s q-L(y) b & s Q
\end{array}\right],\left[\begin{array}{c}
0 \\
\tilde{L}(y)
\end{array}\right]} \\
{\left[0, \tilde{L}(y)^{T}\right],} & B^{+}
\end{array}\right] \succeq 0\right\}
$$

where $B^{+}$stands for the pseudo-inverse of $B$.

It is unknown whether one can find an LMI representation for the set $S_{\mathrm{D}}^{o}$, where $S$ is a second order cone in $\mathcal{X}, \mathrm{D}$ is a second order cone in $\mathcal{W}$, and the bilinear mapping is general. According to Proposition 2.5, this is equivalent to the question whether the following bi-positive set has an LMI representation or not:

$$
\mathcal{B}(C, D)=\left\{Z \mid u^{T} Z v \geq 0 \text { for all } u \in C, v \in D\right\}
$$

where $C$ and $D$ are two second order cones.

In general, let $0 \in S \subseteq \Re^{n}$ be an arbitrary convex set. For $\tau>0$ we denote

$$
\tau S=\{x \mid x / \tau \in S\} .
$$

Then, obviously, $(\tau S)_{\mathrm{D}}^{o}=\frac{1}{\tau}(S)_{\mathrm{D}}^{o}$. Similarly, if $0 \in \mathrm{D}$ then $(S)_{\tau \mathrm{D}}^{o}=\tau(S)_{\mathrm{D}}^{o}$.

Proposition 4.4 Suppose that $0 \in \operatorname{int}\left(E_{1}\right) \subseteq S \subseteq \tau E_{1} \subseteq \Re^{n}$ and $0 \in \operatorname{int}\left(E_{2}\right)$ $\subseteq \mathrm{D} \subseteq \kappa E_{2} \subseteq \Re^{k}$, where $E_{1}$ and $E_{2}$ are two convex sets. It holds that

$$
\frac{1}{\tau}\left(E_{1}\right)_{E_{2}}^{o} \subseteq(S)_{\mathrm{D}}^{o} \subseteq \kappa\left(E_{1}\right)_{E_{2}}^{o} .
$$


One implication of the above proposition is the following. Although it may be difficult to exactly compute the D-induced dual sets, it is possible to approximate it with ellipsoidal duals, which have been shown to be LMI representable (Theorem 4.2). According to the Löwner-John theorem (see e.g. Theorem 3.1.9 of [4]), any full-dimensional and bounded convex set can be approximated by a pair of co-centered ellipsoids (one included in the set and the other including it) with a factor no more than the dimension of the space. This shows that any D-induced polar of a bounded set can be in principle approximated by LMI representable sets with a finite approximation bound. For some special cases, the approximation bounds can be provably better. We shall consider one example to illustrate our point. First note the following results due to Nemirovskii et al. [7], Nesterov [8,9], and Ye [13].

\section{Lemma 4.5 Consider the quadratic optimization problem}

$$
\begin{aligned}
\text { (QP) maximize } & x^{T} P_{0} x \\
\text { subject to } & x^{T} P_{i} x \leq 1, i=1, \ldots, m
\end{aligned}
$$

where $P_{i} \succeq 0, i=0,1, \ldots, m$, and its semidefinite programming $(S D P)$ relaxation

$$
\begin{aligned}
\text { (SDP) maximize } & P_{0} \bullet X \\
\text { subject to } & P_{i} \bullet X \leq 1, i=1, \ldots, m \\
& X \succeq 0 .
\end{aligned}
$$

Suppose that (QP) has an optimal solution. Let $v(\mathrm{QP})$ be the optimal value of (QP) and $v(\mathrm{SDP})$ be the optimal value of (SDP). Then,

(1) ([7]) It holds that

$$
v(\mathrm{QP}) \leq v(\mathrm{SDP}) \leq \lambda v(\mathrm{QP}),
$$

where $\lambda=2 \log (2 m \mu)$ with $\mu=\min \left\{m ; \max _{1 \leq i \leq m} \operatorname{rank} P_{i}\right\}$.

(2) $([8,9,13])$ If the matrices $P_{i}$ 's are mutually commutative, $i=1, \ldots, m$, then

$$
v(\mathrm{QP}) \leq v(\mathrm{SDP}) \leq \frac{\pi}{2} v(\mathrm{QP}) .
$$

Consider $S=\left\{x \mid x^{T} P_{i} x \leq 1, i=1, \ldots, m\right\} \subseteq \mathcal{X}$ with $P_{i} \succeq 0$ for all $i$ and $\sum_{i=1}^{m} P_{i} \succ 0$, and $\mathrm{D}=\left\{w \mid w^{T} B w \leq 1\right\} \subseteq \mathcal{W}$ with $B \succ 0$. The bilinear mapping is given as (14). It is clear that

$$
(S)_{\mathrm{D}}^{o}=\left\{y \mid x^{T}\left(L(y) B L(y)^{T}\right) x \leq 1 \text { for all } x^{T} P_{i} x \leq 1, i=1, \ldots, m\right\} .
$$

Checking the membership for the above set is NP-hard in general. Consider now an approximation using the SDP relaxation

$$
A\left(S_{\mathrm{D}}^{o}\right):=\left\{y \mid\left(L(y) B L(y)^{T}\right) \bullet X \leq 1 \text { for all } P_{i} \bullet X \leq 1, i=1, \ldots, m, X \succeq 0\right\} .
$$


Clearly, $A\left(S_{\mathrm{D}}^{o}\right) \subseteq(S)_{\mathrm{D}}^{o}$. By the strong duality theorem for SDP under the primal-dual Slater condition, we can rewrite $A\left(S_{\mathrm{D}}^{o}\right)$ as

$$
\begin{aligned}
A\left(S_{\mathrm{D}}^{o}\right) & =\left\{y \mid \exists t_{i} \geq 0, i=1, \ldots, m, \sum_{i=1}^{m} t_{i} P_{i} \succeq L(y) B L(y)^{T}, \sum_{i=1}^{m} t_{i} \leq 1\right\} \\
& =\left\{y \mid \exists t_{i} \geq 0, i=1, \ldots, m,\left[\begin{array}{cc}
\sum_{i=1}^{m} t_{i} P_{i} & L(y) \\
L(y)^{T} & B^{-1}
\end{array}\right] \succeq 0, \sum_{i=1}^{m} t_{i} \leq 1\right\}
\end{aligned}
$$

where the second step is due to the Schur complement lemma, and this represents $A\left(S_{\mathrm{D}}^{o}\right)$ by LMI. Lemma 4.5 asserts that if $y \in(S)_{\mathrm{D}}^{o}$ then $\left(L(y) B L(y)^{T}\right) \bullet$ $X \leq \lambda$ for all $P_{i} \bullet X \leq 1, i=1, \ldots, m, X \succeq 0$, where $\lambda=2 \log (2 m \mu)$ with $\mu=\min \left\{m ; \max _{1 \leq i \leq m} \operatorname{rank} P_{i}\right\}$ for general $P_{i} \succeq 0$, and $\lambda=\pi / 2$ if $P_{i}$ 's are mutually commutative. Since $L(y)$ is linear in $y$, it follows that $y / \sqrt{\lambda} \in A\left(S_{\mathrm{D}}^{o}\right)$. Therefore, in this case we have

$$
A\left(S_{\mathrm{D}}^{o}\right) \subseteq(S)_{\mathrm{D}}^{o} \subseteq \sqrt{\lambda} A\left(S_{\mathrm{D}}^{o}\right)
$$

\section{Applications}

To appreciate how the D-induced duality helps to model and solve optimization problems, we shall first discuss two examples of application in this section, before moving on to discuss more theoretical properties of the $\mathrm{D}$-induced dual cones.

\subsection{Robust optimization}

The notion of robust optimization was studied by Ben Tal and Nemirovskii in [1]. Let us now formulate the robust optimization problem using the newly introduced notion of D-induced duality. Consider a general convex conic optimization problem as follows:

$$
\begin{aligned}
& \text { (P) minimize } \quad c^{T} x \\
& \text { subject to } A x+b \in \mathrm{D}
\end{aligned}
$$

or equivalently,

$$
\begin{array}{cl}
\text { (P) } \operatorname{minimize} & c^{T} x \\
\text { subject to } & A x+b x_{0} \in \mathrm{D} \\
& x_{0}=1,
\end{array}
$$

where $\mathrm{D} \subseteq \Re^{k}$ is a given closed convex cone.

The cone $\mathrm{D}$ can be the first orthant (linear programming), or the product of second order cones (second order cone programming), or the cone of positive 
semidefinite matrices (semidefinite programming). In [14] or [2], it is shown that a standard convex program can also be formulated in the conic form in a natural way.

The issue of robust optimization arises when the data of the problem, $A$ and $b$ in the above formulation, are uncertain. In other words, they are perturbed by noises, or 'polluted'. Note that the objective vector $c$ can always be assumed to be certain. This is achieved by reformulating the problem as follows. We introduce one additional variable, $x_{n+1}$, and one additional constraint, $c^{T} x-x_{n+1}=0$, the cone is changed to $\mathcal{K} \times \Re$, and the objective is changed to minimize $x_{n+1}$. For convenience we assume that in the original problem, the objective vector $c$ is already certain.

In many applications, it is crucial to ensure the robustness of the decision $x$. One way to do this is to guarantee that $x$ should remain feasible for all possible data within an 'uncertainty set'.

Let $A_{0}$ and $b_{0}$ be the nominal value of the data. The general structure of the 'polluted' data set is assumed to be

$$
A=A_{0}+\sum_{i=1}^{m}\left(\sum_{j=1}^{k_{i}} u_{j}^{i} A_{j}^{i}\right) \quad \text { and } \quad b=b_{0}+\sum_{i=1}^{m}\left(\sum_{j=1}^{k_{i}} u_{j}^{i} b_{j}^{i}\right),
$$

where $u^{i}=\left[u_{1}^{i}, \ldots, u_{k_{i}}^{i}\right]^{T} \in U^{i} \subseteq \Re^{k_{i}}$, and $U^{i}$ is known as the uncertainty region, $i=1, \ldots, m$. The matrices $A_{j}^{i} \in \Re^{k \times n}$ and vectors $b_{j}^{i} \in \Re^{k}$ are used to model the structure of the uncertainty.

A robustly feasible solution for $(P), x$, will need to satisfy $A x+b \in \mathrm{D}$ for all data $A$ and $b$ as specified in (19) where $u^{i} \in U^{i}, i=1, \ldots, m$.

Let $\mathcal{X}=\Re^{n+1}, \mathcal{Y}=\Re^{k \times(n+1)}$, and $\mathcal{W}=\Re^{k}$. For $\left[x_{0}, x^{T}\right]^{T} \in \mathcal{X}$ and $y=$ $\operatorname{vec}\left(\left[y_{0}, Y\right]\right) \in \mathcal{Y}$ with $y_{0} \in \Re^{k}$ and $Y \in \Re^{k \times n}$, introduce the bilinear mapping as follows:

$$
\left\langle\left[\begin{array}{c}
x_{0} \\
x
\end{array}\right], \operatorname{vec}\left(\left[y_{0}, Y\right]\right)\right\rangle:=\left[y_{0}, Y\right]\left[\begin{array}{c}
x_{0} \\
x
\end{array}\right] .
$$

Let $k_{0}=1$ and $U^{0}=\{1\}, A_{1}^{0}=A_{0}$, and $b_{1}^{0}=b_{0}$. Let

$$
S^{i}:=\left\{\left[\sum_{j=1}^{k_{i}} u_{j}^{i} b_{j}^{i}, \sum_{j=1}^{k_{i}} u_{j}^{i} A_{j}^{i}\right] \mid u^{i} \in U^{i}\right\}, \quad i=0,1, \ldots, m,
$$

where $S^{0}=\left[b_{0}, A_{0}\right]$ by convention. The uncertainty set $S^{i} \subseteq \mathcal{Y}$ is a linear image of $U^{i}, i=1, \ldots, m$. In particular, if $U^{i}$ is a polyhedron/ellipsoid/LMI representable set, then $S^{i}$ is also a polyhedron/ellipsoid/LMI representable set respectively.

A solution $x$ is feasible for $(P)$ in the robust sense if and only if $\left\langle\left[\begin{array}{c}x_{0} \\ x\end{array}\right], \operatorname{vec}\left(\left[y_{0}, Y\right]\right)\right\rangle \in \mathrm{D}$ for all $\left[y_{0}, Y\right] \in \sum_{i=0}^{m} S^{i}$. Because $S^{0}$ is a singleton, 
using Proposition 3.3 we obtain

$$
\left[\begin{array}{l}
1 \\
x
\end{array}\right] \in\left(\sum_{i=0}^{m} S^{i}\right)_{\mathrm{D}}^{o}=\left(S^{0}\right)_{\mathrm{D}}^{o} \cap\left(\bigcap_{i=1}^{m}\left(S^{i}\right)_{\mathrm{D}}^{o}\right) .
$$

The robust optimization version of $(P)$ is

$$
\begin{aligned}
& \text { (RP) minimize } c^{T} x \\
& \text { subject to }\left[\begin{array}{l}
1 \\
x
\end{array}\right] \in\left(\sum_{i=0}^{m} S^{i}\right)_{\mathrm{D}}^{o}=\left(S^{0}\right)_{\mathrm{D}}^{o} \cap\left(\bigcap_{i=1}^{m}\left(S^{i}\right)_{\mathrm{D}}^{o}\right) \text {. }
\end{aligned}
$$

Note that $\left\{x \mid\left[1, x^{T}\right]^{T} \in\left(S^{0}\right)_{\mathrm{D}}^{o}\right\}$ is the original non-robust feasible set. The structure of the constraint in (RP) due to the robustness consideration is apparent in this formulation.

Some well-known results in robust optimization (see [1]) follow immediately from this observation.

For robust linear programming, i.e., $\mathrm{D}=\Re_{+}^{n}$, if the uncertainty set $S^{i}$ is a polyhedron/ellipsoid/LMI representable set, then $\left(S^{i}\right)_{\mathrm{D}}^{o}$ is a polyhedron/ellipsoid/LMI representable set respectively, and so the robust counter part (RP) is a linear program/SOC program (second order cone program)/SDP program (semidefinite program), respectively.

For robust Second Order Cone Programming, i.e., $\mathrm{D}=\mathrm{SOC}(n)$, if the uncertainty set $S^{i}$ is a polyhedron, then $\left(S^{i}\right)_{\mathrm{D}}^{o}$ is an intersection of second order cones; hence the robust counter part (RP) remains an SOC problem. If the uncertainty set $S^{i}$ is an ellipsoid, then it remains an open question whether $\left(S^{i}\right)_{\mathrm{D}}^{o}$ is LMI representable or not. Our conjecture is yes. If so, then the corresponding (RP) is an SDP problem. But even if the answer is no, one still has a polynomial time procedure for checking the membership of the convex set $\left(S^{i}\right)_{\mathrm{D}}^{o}$. By the ellipsoid method, the robust counter part (RP) can be solved efficiently in principle. If $S^{i}$ is a general LMI representable set, then $\left(S^{i}\right)_{\mathrm{D}}^{o}$ is intractable, since this is already so when $S^{i}$ is given in the form of a polyhedron (linear inequalities) rather than in the form of a polytope (convex combinations of vertices).

For robust Semidefinite Programming, i.e., $\mathrm{D}=\mathcal{S}_{+}^{m}$, the robust set $\left(S^{i}\right)_{\mathrm{D}}^{o}$ is intractable when $S^{i}$ is an ellipsoid or an LMI representable set (see e.g. Proposition 4.1); it is tractable if the uncertainty set $S^{i}$ is a polytope, when (RP) remains a semidefinite program.

An interesting case is the robust least square problem studied by El Ghaoui and Lebret [3]:

$$
\begin{array}{ll}
\operatorname{minimize} & t \\
\text { subject to } & t \geq(A x-b)^{T}(A x-b),[A, b] \in \text { 'uncertainty set'. }
\end{array}
$$


To put the problem into the perspective of the D-induced duality, let us introduce

$$
\bar{x}:=\left[\begin{array}{c}
x_{0} \\
t \\
x
\end{array}\right] \quad \text { and } \quad \bar{y}:=\left[\begin{array}{c}
s \\
y_{0} \\
\operatorname{vec}(Y)
\end{array}\right]
$$

and the bilinear mapping

$$
\langle\bar{x}, \bar{y}\rangle:=\left[\begin{array}{c}
t s \\
Y x-x_{0} y_{0}
\end{array}\right]=:\left[\begin{array}{c}
w_{0} \\
w
\end{array}\right] \in \mathcal{W} .
$$

Let $S:=\left\{\bar{y} \mid s=1, y_{0}=b, Y=A\right.$, and $[A, b] \in$ 'uncertainty set' $\}$. Let $\mathrm{D}=$ $\left\{\left[w_{0}, w^{T}\right]^{T} \mid w_{0} \geq w^{T} w\right\}$. According to Corollary 4.3, the set $(S)_{\mathrm{D}}^{o}$ is LMI representable if the uncertainty set is a convex hull of a finite number of ellipsoids. The robust version of the least square problem can thus be formulated as

$$
\begin{array}{ll}
\operatorname{minimize} & t \\
\text { subject to } & {\left[\begin{array}{l}
1 \\
t \\
x
\end{array}\right] \in(S)_{\mathrm{D}}^{o},}
\end{array}
$$

which turns out to be an SDP problem in that case (cf. Proposition 3.3 and Corollary 4.3).

\subsection{Multiple objective conic programming}

Let us consider the following ordering relation based on a convex cone $\mathrm{D} \subseteq \mathfrak{R}^{k}$ :

$$
x \succeq_{\mathrm{D}} y \quad \text { if and only if } \quad x-y \in \mathrm{D} .
$$

The multiple objective convex conic program is now given as

$$
\begin{array}{lll}
(\mathrm{P})_{\mathrm{D}} & \min _{\mathrm{D}} & C x \\
& \text { s.t. } & A x=b \\
& & x \in \mathcal{K}
\end{array}
$$

where $C \in \Re^{k \times n}, A \in \Re^{m \times n}, b \in \Re^{m}$ and $\mathcal{K} \subseteq \Re^{n}$ is a convex cone. Its dual problem can be derived by means of the Lagrangian multipliers and lower bounding. Namely, we obtain the dual problem by attempting to establish a lower bound for the objective in $(\mathrm{P})_{\mathrm{D}}$. Let $Y$ be a linear mapping $\Re^{m} \rightarrow \Re^{k}$ to be applied on both sides of $A x=b$, leading to $Y A x=Y b$. In order for $Y A x$ to be a lower bound for the objective vector, we need to have $(C-Y A) x \in \mathrm{D}$ for all primal feasible $x$. 
Let us consider the bilinear mapping defined as

$$
\langle x, S\rangle=S x \in \Re^{k} .
$$

Clearly, the above bilinear mapping is dual surjective, because $L(S)=S^{T}$ and so the equation $L(S)=a b^{T}$ is always solvable.

In the notion of the D-induced duality, the condition $(C-Y A) x \in \mathrm{D}$ for all $x \in \mathcal{K}$ is simply $C-Y A \in \mathcal{K}_{\mathrm{D}}^{*}$. Now we wish to optimize over all the bounds obtained this way. This naturally leads to the dual problem given as follows:

$$
\begin{array}{lll}
\text { (D) } \mathrm{D} & \max _{\mathrm{D}} & Y b \\
& \text { s.t. } & Y A+S=C \\
& & S \in \mathcal{K}_{\mathrm{D}}^{*}
\end{array}
$$

with respect to the bilinear mapping $\Re^{n} \times \mathfrak{R}^{k \times n} \rightarrow \mathfrak{R}^{k}$ given by $\langle x, S\rangle=S x$. Denote $F_{P}$ to be the feasible set for $(\mathrm{P})_{\mathrm{D}}$ and $F_{D}$ to be the feasible set for $(\mathrm{D})_{\mathrm{D}}$.

By this construction, and also using the extended bipolar theorem, Theorem 2.4, we have the following result, an analog of the weak duality theorem.

Theorem 5.1 For any $x \in F_{P}$ and $(Y, S) \in F_{D}$, we have

$$
C x \succeq \mathrm{D} Y b .
$$

Moreover, if $\mathrm{D}$ and $\mathcal{K}$ are closed convex cones then the dual of $(\mathrm{D})_{\mathrm{D}}$, with respect to the bilinear mapping $\Re^{n} \times \Re^{k \times n} \rightarrow \Re^{k}$ given by $\langle x, S\rangle=S x$, is precisely $(\mathrm{P})_{\mathrm{D}}$ again.

We should note that the D-induced duality is based on the choice of the bilinear mapping. Therefore, if $(\mathrm{D})_{D}$ would be considered as a primal problem, then its dual, taken with respect to the bilinear mapping $\mathfrak{R}^{k \times n} \times \mathfrak{R}^{k \times(k \times n)} \rightarrow \mathfrak{R}^{k}$, is not $(\mathrm{P})_{\mathrm{D}}$ but another problem in a larger space.

It is obvious that if $(\mathrm{P})_{\mathrm{D}}$ is a multi-objective linear program, i.e., both $\mathcal{K}=\Re_{+}^{n}$ and $\mathrm{D}=\mathfrak{R}_{+}^{k}$, then $(\mathrm{D})_{\mathrm{D}}$ is also a multi-objective linear program. In particular, if the primal problem is

$$
\begin{array}{lll}
(\mathrm{P})_{\mathrm{D}} & \min _{\mathfrak{R}_{+}^{k}} & C x \\
& \text { s.t. } & A x=b \\
& & x \geq 0,
\end{array}
$$

then its dual problem is

$$
\begin{array}{lll}
\text { (D) } \mathrm{D} & \max _{\Re_{+}^{k}} & Y b \\
& \text { s.t. } & Y A \leq C .
\end{array}
$$

It is well known that, if the ordering ' $\succeq_{D}$ ' is incomplete, there might be multiple, incomparable, optimal solutions from either the primal or dual point of view, known as the Pareto optimal solutions. 
In general, if $\mathrm{D}$ is a closed cone, then

$$
\mathcal{K}_{\mathrm{D}}^{*}=\{S \mid S x \in \mathrm{D}, \forall x \in \mathcal{K}\}=\mathcal{B}\left(\mathrm{D}^{*}, \mathcal{K}\right) .
$$

If we insist on the strong duality (complementarity), then the optimality can be defined as follows.

Definition 5.2 We call $x^{*}\left(\left(Y^{*}, S^{*}\right)\right)$ a global primal (dual) optimal solution if $C x-C x^{*} \in \mathrm{D}$ for all $x \in F_{P}\left(Y^{*} b-Y b \in \mathrm{D}\right.$ for all $\left.(Y, S) \in F_{D}\right)$.

We call $x^{*}$ and $\left(Y^{*}, S^{*}\right)$ complementary optimal solutions for $(\mathrm{P})_{\mathrm{D}}$ and $(\mathrm{D})_{\mathrm{D}}$, respectively, if they are feasible and $C x^{*}=Y^{*} b$ or equivalently, $S^{*} x^{*}=0 \in \mathrm{D}$.

Obviously, the complementary optimality is a stringent condition: a complementary optimal solution pair must be globally primal and dual optimal solutions, respectively.

Consider the following simple multiple objective linear program:

$$
\begin{array}{lll}
\left(\mathrm{P}_{1}\right)_{\mathrm{D}} \quad \min _{\mathrm{D}} & {\left[\begin{array}{l}
x_{1} \\
x_{2}
\end{array}\right]} \\
\text { s.t. } \quad & x_{1}+x_{2}=1 \\
& {\left[\begin{array}{l}
x_{1} \\
x_{2}
\end{array}\right] \in \Re_{+}^{2} .}
\end{array}
$$

Let us first consider the case where $\mathrm{D}=\Re_{+}^{2}$. Due to the binding relation, no feasible solution of $\left(P_{1}\right)_{D}$ can be dominated by any other feasible solutions. Therefore, they are all Pareto optimal. However, from the complementary optimality point of view, no dominating optimal solution exists. To see this, consider its dual problem

$$
\begin{aligned}
\left(\mathrm{D}_{1}\right)_{\mathrm{D}} \max _{\mathrm{D}} & {\left[\begin{array}{l}
y_{1} \\
y_{2}
\end{array}\right] } \\
\text { s.t. } & {\left[\begin{array}{l}
y_{1} \\
y_{2}
\end{array}\right][1,1]+S=\left[\begin{array}{ll}
1 & 0 \\
0 & 1
\end{array}\right] } \\
& S \in\left(\Re_{+}^{2}\right)_{\mathrm{D}}^{*},
\end{aligned}
$$

with $\mathrm{D}=\mathfrak{R}_{+}^{2}$, or

$$
\begin{array}{rll}
\left(\mathrm{D}_{1}\right)_{\mathrm{D}} & \max _{\mathrm{D}} & {\left[\begin{array}{l}
y_{1} \\
y_{2}
\end{array}\right]} \\
& \text { s.t. } & y_{1} \leq 0, y_{2} \leq 0 .
\end{array}
$$


Obviously the equation $S x=\left[\begin{array}{l}0 \\ 0\end{array}\right]$ cannot have a solution among feasible $x$ and $S$, i.e., no complementary optimal solution exists. However, $\left(D_{1}\right)_{\mathrm{D}}$ does have a globally optimal solution, namely $\left[y_{1}^{*}, y_{2}^{*}\right]=[0,0]$. It turns out that this is no coincidence, as the following theorem reveals.

Theorem 5.3 Suppose that a multi-objective linear program $\left(P_{\mathfrak{R}_{+}^{k}}\right)$ is feasible and has a Pareto optimal solution. Then its dual problem, $\left(D_{\Re_{+}^{k}}\right)$, always has a globally optimal solution.

Proof Observe that the dual problem can be separated in the following way. By letting $Y=\left[y_{1}, \ldots, y_{k}\right]^{T}$ and $C=\left[c_{1}, \ldots, c_{k}\right]^{T}$, and solving $k$ linear programs, max $y_{i}^{T} b$ subject to $y_{i}^{T} A \leq c_{i}^{T}$, with optimal solution $\left(y^{*}\right)_{i}^{T}, i=1, \ldots, k$, one concludes that the optimal solution for $\left(D_{\Re_{+}^{k}}\right)$ is simply $Y^{*}=\left[y_{1}^{*}, \ldots, y_{k}^{*}\right]^{T}$. It remains to argue that all these linear programs must have optimal solutions. First, these must all be feasible, because if $y_{i}^{T} A \leq c_{i}^{T}$ is infeasible for some $i$, then by the Farkas lemma there is $x \geq 0$ such that $A x=0$ and $c_{i}^{T} x<0$, contradicting with the condition that $\left(P_{\Re_{+}^{k}}\right)$ has a Pareto optimal solution. Second, they must be bounded, for otherwise there should be no $x \geq 0$ such that $A x=b$, contradicting with the condition that $\left(P_{\mathfrak{R}_{+}^{k}}\right)$ is feasible. This concludes the theorem.

One can easily extend the result to the situation where $D=\Re_{+}^{k}$ and $\mathcal{K}$ is an arbitrary convex cone. In that case, observe that

$$
\begin{aligned}
(\mathcal{K})_{\mathrm{D}}^{*} & =\left\{S=\left[s_{1}, \ldots, s_{k}\right]^{T} \mid s_{i}^{T} x \geq 0 \forall x \in \mathcal{K}, i=1, \ldots, k\right\} \\
& =\left\{S=\left[s_{1}, \ldots, s_{k}\right]^{T} \mid s_{i} \in \mathcal{K}^{*}, i=1, \ldots, k\right\},
\end{aligned}
$$

and the following extension of Theorem 5.3 can be proved using almost identical arguments, with the extra precaution that one requires the Slater condition for the strong duality; for discussions on the conic duality theory, see [5].

Theorem 5.4 Suppose that a multi-objective conic convex program $\left(P_{\mathfrak{R}_{+}^{k}}\right)$ is strongly feasible, namely there is $x \in$ int $\mathcal{K}$ with $A x=b$. Moreover, suppose that its dual problem, $\left(D_{\mathfrak{R}_{+}^{k}}\right)$, is also strongly feasible, namely there is $Y$ such that $C-Y A \in \operatorname{int}(\mathcal{K})_{\mathfrak{R}_{+}^{k}}^{*}$. Then, $\left(D_{\mathfrak{R}_{+}^{k}}\right)$ has a globally optimal solution.

Theorem 5.4 has an interesting geometric interpretation. Although $\left(\mathrm{P}_{\mathrm{D}}\right)$ may not have any dominating global optimal solution, there is an infeasible point (the dual global optimal solution) that uniformly dominates all the optimal solutions, and yet it is 'closest to feasibility'. That solution is of special interest and can be viewed as a desirable reference point. Among all Pareto points, one may, for instance, choose the one that is closest to that reference point (projection). 
For the problem instance $\left(\mathrm{P}_{1}\right)_{\mathrm{D}}$, let us now consider the conic ordering induced by the lexicographic ordering of the coordinates. In this case, since the lexicographic ordering is complete and the feasible set of $\left(\mathrm{P}_{1}\right)_{D}$ is compact, an optimal solution for $\left(\mathrm{P}_{1}\right)_{D}$ exists. In particular, the optimal solution for $\left(\mathrm{P}_{1}\right)_{D}$ is obviously $\left[x_{1}^{*}, x_{2}^{*}\right]=[0,1]$. Furthermore, we shall see below that in fact a pair of primal-dual complementary optimal solutions exists.

In the two-dimensional case, the lexicographic ordering on $\left(x_{1}, x_{2}\right)$ corresponds to the conic ordering which is defined by the cone

$$
\mathbf{D}=\left\{\left[\begin{array}{l}
x_{1} \\
x_{2}
\end{array}\right] \mid x_{1}>0\right\} \cup\left(\Re_{+}\left[\begin{array}{l}
0 \\
1
\end{array}\right]\right)
$$

which is a non-flat and non-closed convex cone.

Let us compute the cone $\left(\Re_{+}^{2}\right)_{\mathrm{D}}^{*}$. Take an arbitrary $S \in\left(\Re_{+}^{2}\right)_{\mathrm{D}}^{*}$, that is $S x \in \mathrm{D}$ for all $x \in \Re_{+}^{2}$. In other words, $s_{11} x_{1}+s_{12} x_{2} \geq 0$ for all $x_{1}, x_{2} \geq 0$, and if $s_{11} x_{1}+s_{12} x_{2}=0$ then $s_{21} x_{1}+s_{22} x_{2} \geq 0$. This leads to

$$
\left(\Re_{+}^{2}\right)_{\mathrm{D}}^{*}=\left\{\left[\begin{array}{ll}
s_{11} & s_{12} \\
s_{21} & s_{22}
\end{array}\right] \mid \begin{array}{l}
s_{11}, s_{12}>0, \text { or } s_{11}=0, s_{12}>0, s_{21} \geq 0, \\
\text { or } s_{11}>0, s_{12}=0, s_{22} \geq 0, \text { or } s_{11}=s_{12}=0, s_{21} \geq 0, s_{22} \geq 0
\end{array}\right\} .
$$

The dual problem $\left(\mathrm{D}_{1}\right)_{\mathrm{D}}$, where $\mathrm{D}$ is given as in $(21)$, has an optimal solution $y_{1}^{*}=0, y_{2}^{*}=1$, and $S^{*}=\left[\begin{array}{cc}1 & 0 \\ -1 & 0\end{array}\right]$. Moreover, this optimal solution is complementary to the primal optimal solution $\left[x_{1}^{*}, x_{2}^{*}\right]=[0,1]$ as $S^{*} x^{*}=\left[\begin{array}{l}0 \\ 0\end{array}\right]$. A remarkable fact is that the feasible set of $\left(D_{1}\right)_{D}$ is not closed.

\section{Sublinear function representation of convex cones and their tensor product}

In the previous section we have seen some applications of the newly introduced $\mathrm{D}$-induced duality. We also recognized that computing the $\mathrm{D}$-induced dual cone or polar set can be a difficult task in general; see Proposition 4.1. In Sect. 4 we focused on LMI representable D-induced dual objects, and the approximation of them. The aim of this section is to view the computation of the D-induced dual cones from a totally different angle: sublinear function representations of convex cones and their tensor products. This approach allows us another point of view on the difficulty of computing the $\mathrm{D}$-induced dual of a cone.

As Proposition 2.5 asserts, we have for D closed

$$
\begin{aligned}
U_{\mathrm{D}}^{*} & =\left\{y \mid L(y) \in \mathcal{B}\left(U, \mathrm{D}^{*}\right)\right\} \\
& =\left\{y \mid L(y) \in\left(U \otimes \mathrm{D}^{*}\right)^{*}\right\} .
\end{aligned}
$$

It is therefore clear that it is crucial to analyze the dual (in the usual sense) of the tensor product (or the Kronecker product in the matrix form) of two convex cones, if we wish to characterize the D-induced dual cone. 
In this section, we will provide an interpretation of the dual of tensor product of cones that can be represented by a positive sublinear function. To this end, we first show that the computation of the dual of the of tensor product of any two convex cones can be reduced to the computation of the dual of two appropriately defined closed, solid, pointed convex cones. Next, we demonstrate that any closed, solid, pointed convex cone is representable by a positive sublinear function, and finally, we identify the dual cone of the tensor product of thus represented cones. We will reduce this task in two steps to the task of calculating the dual of the tensor product of two cones that are representable by sublinear functions.

First step. The first step is the reduction to closed, solid (that is, full-dimensional), pointed (that is, containing no line through the origin) cones. Let $C \subseteq \mathcal{X}$ and $D \subseteq \mathcal{W}$ be convex cones.

Reduction to closed cones. The reduction to closed cones is achieved by the following equality $(C \otimes D)^{*}=(\operatorname{cl} C \otimes \mathrm{cl} D)^{*}$.

Reduction to pointed cones. Now we will give the reduction to cones that are moreover pointed. Not necessarily pointed cones $C$ and $D$ can be decomposed as follows:

$$
C=P_{c}+L_{c}, \quad \text { and } \quad D=P_{d}+L_{d}
$$

where $P_{c}$ and $P_{d}$ are pointed cones and $L_{p}$ and $L_{d}$ are linear subspaces. To be explicit: $L_{c}=C \cap(-C)$ (resp. $L_{d}=D \cap(-D)$ ), the largest linear subspace contained in $C$ (resp. in $D)$, and $P_{c}$ (resp. $\left.P_{d}\right)$ can be chosen to be $\left\{x \in C:\langle x, \bar{x}\rangle_{\mathcal{X}}=0 \forall \bar{x} \in L_{c}\right\}$ (resp. $\left\{x \in D:\langle w, \bar{w}\rangle_{\mathcal{W}}=0 \forall \bar{w} \in L_{d}\right\}$ ). The promised reduction to pointed cones follows from the equality

$$
C \otimes D=P_{c} \otimes P_{d}+P_{c} \otimes L_{d}+L_{c} \otimes P_{d}+L_{c} \otimes L_{d}
$$

in combination with the following lemma.

Lemma 6.1 Let $K \in \mathcal{X}$ be a convex cone and $L$ a vector space. Then $K \otimes L$ is a linear space, in fact $K \otimes L=(\operatorname{span} K) \otimes L$.

Proof Consider any $\sum_{i} x_{i} y_{i}^{T} \in K \otimes L$ with $x_{i} \in K$ and $y_{i} \in L$ for all $i$. Since $L$ is a linear space, we have

$$
-\sum_{i} x_{i} y_{i}^{T}=\sum_{i} x_{i}\left(-y_{i}\right)^{T} \in K \otimes L
$$

Therefore, this concludes that $K \otimes L$ is a linear space, and furthermore $K \otimes L=$ $(\operatorname{span} K) \otimes L$. This proves the lemma.

Reduction to solid cones. Now it is necessary to indicate the dependence of the ordinary dual of a given cone on the space containing this given cone in the notation. We will write $(E, \mathcal{Z})^{*}$-instead of just $C^{*}$-for the ordinary dual of a 
cone $E$ in a vector space $\mathcal{Z}$. Write

$$
\mathcal{X}=\operatorname{Span} C \oplus(\operatorname{Span} C)^{\perp} \quad \text { and } \quad \mathcal{W}=\operatorname{Span} D \oplus(\operatorname{Span} D)^{\perp}
$$

This leads readily to the conclusion that to determine the dual cone of $C \otimes D$, we may reduce to the case that $C$ and $D$ are solid cones.

We have shown that to compute the dual of the tensor product of $C$ and $D$, it is sufficient to consider the tensor product of cones as defined in the above derivation. This finishes the first step of the reduction.

Second step. The second step is the reduction from closed, solid, pointed convex cones to positive sublinear functions. We need some definitions. We recall that a function $\phi$ on a vector space $\mathcal{U}$ is called sublinear if it satisfies the following conditions:

$$
\phi(t u)=t \phi(u) \quad \text { and } \quad \phi(u+v) \leq \phi(u)+\phi(v)
$$

for all $t \geq 0$ and $u, v \in \mathcal{V}$. This is equivalent to requiring that the epigraph of $\phi$, that is,

$$
\operatorname{epi}(\phi)=\left\{\left[\begin{array}{c}
\phi(u)+r \\
u
\end{array}\right] \mid r \in \Re_{+}, u \in \mathcal{U}\right\},
$$

is a convex cone. We note that the epigraph of a sublinear function $\phi$ on $\mathcal{X}$ is a closed, solid, pointed convex cone. A sublinear function $\phi$ on $\mathcal{U}$ is positive if $\phi(u)>0$ for all nonzero $u$. We call two convex cones $E \subseteq \mathcal{Y}$ and $F \subseteq \mathcal{Z}$ equivalent if there exists a linear bijective transformation $\mathcal{Y} \rightarrow \mathcal{Z}$ which maps $E$ bijectively onto $F$. We say that a convex cone $E \subseteq \mathcal{Y}$ is represented by a sublinear mapping $\phi$ on $\mathcal{U}$ if the convex cones $E \subseteq \mathcal{Y}$ and epi $(\phi) \subseteq \Re \times \mathcal{U}$ are equivalent. Now we can formulate and so carry out the second step as follows.

Proposition 6.2 Each closed, solid, pointed convex cone $C$ can be represented by a positive sublinear function $\phi$.

Proof One has $C^{*} \neq\{0\}$, by separation of convex sets, and so one can choose a nonzero element $y \in C^{*}$. Let $\mathcal{U}=\{x \in \mathcal{X}:\langle x, y\rangle=0\}$ and define the function $\phi$ on $\mathcal{U}$ by

$$
\phi(u)=\inf \{\rho \mid u+\rho y \in C\} .
$$

It is readily verified that $\phi$ is a positive, sublinear function that represents $C$.

For instance, if $C$ is a second order cone, then the corresponding sublinear function can be simply the Euclidean norm $\phi(u)=\|u\|$; if $C$ is an orthant, then $\phi(u)=\max \left\{\max _{i} u_{i}, 0\right\}$; if $C$ is the cone of positive semidefinite matrices, then $\phi(u)=\max \left\{\lambda_{\max }(\operatorname{Mat}(u)), 0\right\}$, where $' \operatorname{Mat}(u)$ ' stands for the stacking operation 
to create a symmetric matrix from $u$, and ' $\lambda_{\max }(X)$ ' stands for the maximum eigenvalue of $X$.

Similarly, if $D$ is another closed solid pointed cone, let us assume that

$$
D=\left\{\left[\begin{array}{c}
\psi(w)+s \\
w
\end{array}\right] \mid s \in \Re_{+}, w \in \mathcal{W}\right\}
$$

where $\mathcal{W}$ is a vector space and $\psi$ is a positive sublinear function on $\mathcal{W}$.

One may be tempted to conjecture that

$$
(C \otimes D)^{*}=C^{*} \otimes D^{*}
$$

This would reduce the problem to that of computing tensor products of cones, in view of the following well-known formula:

$$
\left(C_{\phi}\right)^{*}=C_{\phi^{*}}
$$

where $\phi^{*}(x)=\sup _{x \neq 0} \frac{\langle\bar{x}, x\rangle}{\phi(x)}$ for all $x \in \mathcal{X}$. We mention in passing that this formula gives an explicit demonstration of the fact that the property of cones to be representable by positive sublinear functions is preserved under taking duals.

Unfortunately, the conjectured equality above does not hold in general, although it is obvious that

$$
C^{*} \otimes D^{*} \subseteq(C \otimes D)^{*}
$$

As an example, let us consider $C=D=\mathrm{SOC}(n+1)$. Then, by the CauchySchwartz inequality one easily checks that

$$
\left[\begin{array}{cc}
1 & 0 \\
0 & -I
\end{array}\right] \in(\operatorname{SOC}(n+1) \otimes \operatorname{SOC}(n+1))^{*}
$$

However,

$$
\left[\begin{array}{cc}
1 & 0 \\
0 & -I
\end{array}\right] \notin \operatorname{SOC}(n+1)^{*} \otimes \operatorname{SOC}(n+1)^{*}=\operatorname{SOC}(n+1) \otimes \operatorname{SOC}(n+1) .
$$

Note that in the argument above we have used the self-duality of the second order cone.

Now we turn to the computation of the dual of tensor product of convex cones $C$ and $D$. By the reductions above, we may assume that $C=\operatorname{epi}(\phi)$ and $D=\operatorname{epi}(\psi)$, where $\phi$ and $\psi$ are sublinear functions. 
Then, we may explicitly write the cone of the bi-positive mappings as

$$
\begin{aligned}
& \mathcal{B}(C, D)=\left\{\left[\begin{array}{ll}
t & b^{T} \\
a & M
\end{array}\right] \mid\left[\begin{array}{c}
\phi(u)+r \\
u
\end{array}\right]^{T}\left[\begin{array}{ll}
t & b^{T} \\
a & M
\end{array}\right]\left[\begin{array}{c}
\psi(w)+s \\
w
\end{array}\right]\right. \\
&\left.\geq 0, \forall u \in \mathcal{U}, w \in \mathcal{W}, r, s \in \Re_{+}\right\} .
\end{aligned}
$$

We now aim at a procedure to check the membership for the cone $\mathcal{B}(C, D)$. Consider

$$
\left[\begin{array}{ll}
t & b^{T} \\
a & M
\end{array}\right] \in \mathcal{B}(C, D)
$$

By (22) we know that

$$
t(\phi(u)+r)(\psi(w)+s)+(\phi(u)+r) b^{T} w+(\psi(w)+s) a^{T} u+u^{T} M w \geq 0
$$

for all $u \in \mathcal{U}, w \in \mathcal{W}, r \geq 0$ and $s \geq 0$.

It follows immediately from (23) that $t \geq 0$.

If $t=0$ then

$$
(\phi(u)+r) b^{T} w+(\psi(w)+s) a^{T} u+u^{T} M w \geq 0
$$

for all $u \in \mathcal{U}, w \in \mathcal{W}, r \geq 0$ and $s \geq 0$. This leads to $a^{T} u \geq 0$ for all $u \in \mathcal{U}$, and $b^{T} w \geq 0$ for all $w \in \mathcal{W}$. Since $\mathcal{U}$ and $\mathcal{W}$ are vector spaces, we conclude that $a=0$ and $b=0$. Therefore the inequality reduces further to

$$
u^{T} M w \geq 0, \quad \forall u \in \mathcal{U} \quad \text { and } \quad \forall w \in \mathcal{W} .
$$

Since $\mathcal{U}$ and $\mathcal{W}$ are vector spaces, we get $M=0$.

Now we consider the situation $t>0$. Without losing generality let us scale the value of $t$ and assume $t=1$. As $\mathcal{B}(C, D)$ is a cone, the scaling does not change the nature of the membership checking procedure.

We now rewrite (23) as

$$
\begin{aligned}
& (\phi(u)+r)(\psi(w)+s)+(\phi(u)+r) b^{T} w+(\psi(w)+s) a^{T} u+u^{T} M w \\
& =\phi(u) \psi(w)+u^{T} M w+\phi(u) b^{T} w+\psi(w) a^{T} u+\left(\phi(u)+a^{T} u\right) s \\
& \quad+\left(\psi(w)+b^{T} w\right) r+r s \\
& \geq 0
\end{aligned}
$$

for all $u \in \mathcal{U}, w \in \mathcal{W}, r \geq 0$ and $s \geq 0$. It is evident that (24) is equivalent to the following three conditions: 
$\phi(u)+a^{T} u \geq 0 \quad$ for all $u \in \mathcal{U}$,

$\psi(w)+b^{T} w \geq 0$ for all $w \in \mathcal{W}$,

$\phi(u) \psi(w)+u^{T} M w+\phi(u) b^{T} w+\psi(w) a^{T} u \geq 0 \quad$ for all $u \in \mathcal{U}, w \in \mathcal{W}$.

Conditions (25) and (26) are equivalent to

$$
\left[\begin{array}{l}
1 \\
a
\end{array}\right] \in C^{*}
$$

and

$$
\left[\begin{array}{l}
1 \\
b
\end{array}\right] \in D^{*}
$$

Finally, condition (27) is equivalent to the following two statements:

$$
\begin{aligned}
& {\left[\begin{array}{c}
\psi(w)+b^{T} w \\
\psi(w) a+M w
\end{array}\right] \in C^{*} \quad \text { for all } w \in \mathcal{W},} \\
& {\left[\begin{array}{c}
\phi(u)+a^{T} u \\
\phi(u) b+M^{T} u
\end{array}\right] \in D^{*} \quad \text { for all } u \in \mathcal{U} .}
\end{aligned}
$$

We note that it may or may not be a simple task to verify conditions (30) and (31). For instance, when $C$ and $D$ are second order cones, then conditions (30) and (31) can be reduced to verifying

$$
1+b^{T} w \geq\|M w+a\| \quad \text { for all }\|w\|=1
$$

and

$$
1+a^{T} u \geq\left\|M^{T} u+b\right\| \quad \text { for all }\|u\|=1 .
$$

Using the $S$-procedure result once again, this can be achieved by checking the following conditions:

$$
\left\{\begin{array}{l}
b^{T} b-1 \leq 0 \\
\exists \tau:\left[\begin{array}{cc}
1-a^{T} a & b^{T}+a^{T} M \\
b+M^{T} a & b b^{T}-M^{T} M
\end{array}\right]+\tau\left[\begin{array}{cc}
-1 & 0 \\
0 & I
\end{array}\right] \succeq 0, \\
a^{T} a-1 \leq 0 \\
\exists \kappa:\left[\begin{array}{ll}
1-b^{T} b & a^{T}+b^{T} M^{T} \\
a+M b & a a^{T}-M M^{T}
\end{array}\right]+\kappa\left[\begin{array}{cc}
-1 & 0 \\
0 & I
\end{array}\right] \succeq 0 .
\end{array}\right.
$$

These conditions, though expressed as matrix inequalities, are not linear in $a, b$ and $M$.

For general convex cones, however, as Proposition 4.1 reveals, checking membership in D-induced dual cones is a hard task. 


\section{Concluding remarks}

In this paper we have extended the definition of duality using a pre-described conic ordering relation and a bilinear mapping. The new type of dual objects is shown to be useful. It also brings up interesting theoretical questions such as how to characterize the bi-positive cones, and how to compute/approximate the relevant dual objects. A good understanding of this subject appears to be important both for the theory and practice of optimization and for convex analysis in general. As immediate research topics we pose the following two questions: (1) Is it possible to describe using LMI's the cone $(U)_{\mathrm{D}}^{*}$ where $U$ is a second order cone in $\mathcal{X}$ and $\mathrm{D}$ is a second order cone in $\mathcal{W}$ ? As we remarked in Sect. 4, this amounts to the LMI representation of the bi-positive set $\mathcal{B}(C, D)$ where $C$ and $D$ are second order cones. (2) In Sect. 5 we showed that the dual of a multi-objective conic optimization problem, denoted by (D) $)_{D}$, always has an attainable global optimal solution under some suitable Slater type regularity conditions, provided that the multi-objectives are ordered by the first orthant. Does this result remain true for a general order-defining cone $D$ or not?

Acknowledgements We would like to thank the careful referee for helping us to clear the present introduction to new territory from unnecessary obstacles to the reader.

\section{References}

1. Ben-Tal, A., Nemirovskii, A.: Robust convex optimization. Math. Oper. Res. 23, 769-805 (1998)

2. Brinkhuis, J., Tikhomirov, V.: Optimization: insights and applications. Princeton University Press, Princeton (2005)

3. El Ghaoui, L., Lebret, H.: Robust solutions to least-squares problems with uncertain data. SIAM J. Matrix Anal. Appl. 18, 1035-1064 (1997)

4. Grötschel, M., Lovász, L., Schrijver, A.: Geometric Algorithms and Combinatorial Optimization. Springer, Heidelberg (1993)

5. Luo, Z.Q., Sturm, J.F., Zhang, S.: Duality results for conic convex programming. Technical Report 9719/A, Econometric Institute, Erasmus University Rotterdam, The Netherlands (1997)

6. Luo, Z.Q., Sturm, J.F., Zhang, S.: Multivariate nonnegative quadratic mappings. SIAM J. Optim. 14, 1140-1162 (2004)

7. Nemirovskii, A., Roos, C., Terlaky, T.: On maximization of quadratic form over intersection of ellipsoids with common center. Math. Progr. 86, 463-473 (1999)

8. Nesterov, Yu.: Semidefinite relaxation and nonconvex quadratic optimization. Optim. Methods Software 9, 141-160 (1998)

9. Nesterov, Yu.: Global quadratic optimization on the sets with simple structure. Working Paper, Université Catholique de Louvain, Louvain-la-Neuve, Belgium (1999)

10. Nesterov, Yu., Nemirovsky, A.: Interior point polynomial methods in convex programming. Stud. Appl. Math. 13 (1994)

11. Rockafellar, R.T.: Convex Analysis. Princeton University Press, Princeton (1970)

12. Sturm, J.F., Zhang, S.: On cones of nonnegative quadratic functions. Math. Oper. Res. 28, 246267 (2003)

13. Ye, Y.: Approximating quadratic programming with bound and quadratic constraints. Math. Progr. 84, 219-226 (1999)

14. Zhang, S.: A new self-dual embedding method for convex programming. J. Global Optim. 29, 479-496 (2004) 